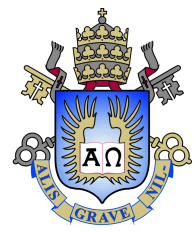

Jéssica Andreza Manghi dos Santos

\title{
Desigualdade de Harnack e Estimativas de Holder para Equações Elípticas de Segunda Ordem
}

Dissertação apresentada como requisito parcial para obtenção do grau de Mestre pelo Programa de Pós-graduação em Matemática, do Departamento de Matemática da PUC-Rio.

Orientador: Prof. Boyan Slavchev Sirakov 


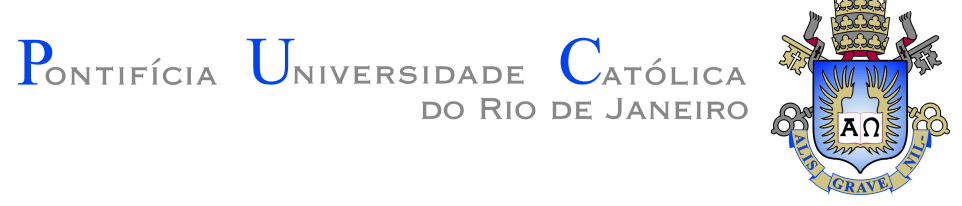

Jéssica Andreza Manghi dos Santos

Desigualdade de Harnack e Estimativas de
Holder para Equações Elípticas de Segunda
Ordem

Dissertação apresentada como requisito parcial para obtenção do grau de Mestre pelo Programa de Pós-graduação em Matemática da PUC-Rio. Aprovada pela Comissão Examinadora abaixo:

Prof. Boyan Slavchev Sirakov

Orientador

Departamento de Matemática - PUC-Rio

Prof. Ricardo José Alonso Plata

Departamento de Matemática - PUC-Rio

Prof. Mauro Antonio Rincon

UFRJ

Prof. Amaury Alvarez Cruz

UFRJ

Rio de Janeiro, 07 de Julho de 2021 
Todos os direitos reservados. A reprodução, total ou parcial do trabalho, é proibida sem a autorização da universidade, do autor e do orientador.

Jéssica Andreza Manghi dos Santos

Graduada em Matemática-Licenciatura na Universidade Federal Fluminense/UFF

Ficha Catalográfica

Manghi dos Santos, Jéssica Andreza

Desigualdade de Harnack e Estimativas de Holder para Equações Elípticas de Segunda Ordem / Jéssica Andreza Manghi dos Santos; orientador: Boyan Slavchev Sirakov. 2021.

59 f: il. color. ; $30 \mathrm{~cm}$

Dissertação (mestrado) - Pontifícia Universidade Católica do Rio de Janeiro, Departamento de Matemática, 2021.

Inclui bibliografia

1. Matemática - Teses. 2. Análise e Equações Diferenciais - Teses. 3. EDP. 4. Equações Diferenciais Parciais. 5. Desigualdade de Harnack. 6. Estimativas de Holder. 7. Equações Elípticas. I. Sirakov, Boyan. II. Pontifícia Universidade Católica do Rio de Janeiro. Departamento de Matemática. III. Título. 


\section{Agradecimentos}

Agradeço em primeiro lugar a Deus, por me permitir alcançar mais um importante passo em minha vida.

Ao professor Boyan Sirakov, minha imensa admiração por seu trabalho e gratidão pela sua orientação.

A minha mãe e ao meu padrasto, Cristina e Roberto, pelo amor incondicional e por serem a força que me faz seguir a diante.

A minha irmã Jennifer, pelo incentivo e apoio em todos os momentos.

A secretaria da pós-graduação por todo suporte prestado.

O presente trabalho foi realizado com apoio da Coordenação de Aperfeiçoamento de Pessoal de Nível Superior - Brasil (CAPES) - Código de Financiamento 001. 


\section{Resumo}

Manghi dos Santos, Jéssica Andreza; Sirakov, Boyan. Desigualdade de Harnack e Estimativas de Holder para Equações Elípticas de Segunda Ordem. Rio de Janeiro, 2021. 59p. Dissertação de Mestrado - Departamento de Matemática, Pontifícia Universidade Católica do Rio de Janeiro.

O objetivo principal dessa dissertação é estudar a desigualdade de Harnack e as estimativas de Holder, para um operador elíptico de segunda ordem, na forma não divergente $(N D)$ e na forma divergente $(D)$, respectivamente,

$$
\begin{gathered}
L=a^{i j}(x) \frac{\partial^{2}}{\partial x^{i} \partial x^{j}}+B^{i}(x) \frac{\partial}{\partial x_{i}}-c(x) \\
L u=D_{i}\left(a^{i j}(x) D_{j} u\right)+b^{i}(x) u+c^{i}(x) D_{i} u+d(x) u
\end{gathered}
$$

sendo os coeficientes $a^{i j}, b^{i}, c^{i}, c, B^{i}, d(i, j=1, \ldots, n)$ funções mensuráveis e limitadas em um domínio $\Omega \subset \mathbb{R}^{n}$.

\section{Palavras-chave}

EDP; Equações Diferenciais Parciais; Desigualdade de Harnack; Estimativas de Holder; Equações Elípticas. 


\section{Abstract}

Manghi dos Santos, Jéssica Andreza; Sirakov, Boyan (Advisor). Harnack's Inequality and Holder Estimates for Second Order Elliptical Equations. Rio de Janeiro, 2021. 59p. Dissertação de Mestrado - Departamento de Matemática, Pontifícia Universidade Católica do Rio de Janeiro.

The main objective of this dissertation is to study Harnack's inequality and Holder's estimates for a second-order elliptical operator, written in the non-divergent form $(N D)$ and in the divergent form $(D)$, respectively,

$$
\begin{gathered}
L=a^{i j}(x) \frac{\partial^{2}}{\partial x^{i} \partial x^{j}}+B^{i}(x) \frac{\partial}{\partial x_{i}}-c(x) \\
L u=D_{i}\left(a^{i j}(x) D_{j} u\right)+b^{i}(x) u+c^{i}(x) D_{i} u+d(x) u
\end{gathered}
$$

where the coefficients $a^{i j}, b^{i}, c^{i}, c, B^{i}, d(i, j=1, \ldots, n)$ are measurable bounded functions in a domain $\Omega \subset \mathbb{R}^{n}$.

\section{Keywords}

PDE; Partial Differential Equations; Harnack's Inequality; Holder Estimate; Elliptical Equation. 


\section{Sumário}

1 Introdução $\quad 8$

2 Resultados Preliminares $\quad 10$

2.0.1 Espaços $L^{p}$. 10

2.0.2 Espaços de Sobolev 12

3 Regularidades de Equações Elípticas na Forma Não Divergente 16

3.3.1 Lemas de Crescimento 24

3.3.2 Desigualdade de Harnack para Equações Elípticas 29

3.3.3 Propriedades de Hölder da Solução da Equação Elíptica 31

4 Regularidades de Equações Elípticas na Forma Divergente 37

4.4.1 Limitações Globais de Soluções Fracas 38

4.4.2 Propriedades Locais de Soluções Fracas 46

4.4.3 Principio do Máximo Forte 53

4.4.4 Desigualdade de Harnack 54

4.4.5 Propriedades de Hölder 54

$\begin{array}{ll}\text { Referências bibliográficas } & 58\end{array}$ 


\section{1 \\ Introdução}

Nesta dissertação estudaremos propriedades de regularidades de soluções fracas para um operador elíptico de segunda ordem, escrito na forma não divergente $(N D)$ e na forma divergente $(D)$, respectivamente,

$$
\begin{gathered}
L=a^{i j}(x) \frac{\partial^{2}}{\partial x^{i} \partial x^{j}}+B^{i}(x) \frac{\partial}{\partial x_{i}}-c(x) \\
L u=D_{i}\left(a^{i j}(x) D_{j} u\right)+b^{i}(x) u+c^{i}(x) D_{i} u+d(x) u
\end{gathered}
$$

sendo os coeficientes $a^{i j}, b^{i}, c^{i}, c, B^{i}, d(i, j=1, \ldots, n)$ funções mensuráveis e limitadas em um domínio $\Omega \subset \mathbb{R}^{n}$.

No caso não divergente os coeficientes satisfazem as seguintes hipóteses:

$$
\lambda|\xi|^{2} \leq a^{i j}(x) \xi^{i} \xi^{j} \leq \lambda^{-1}|\xi|^{2},|B(x)| \leq \lambda^{-1}, 0 \leq c(x) \leq \lambda^{-1}
$$

para algum número real $0<\lambda<1, \xi=\left(\xi^{1}, \ldots, \xi^{n}\right) \in \mathbb{R}^{n}$ onde

$B(x)=\left(B^{1}(x), \ldots, B^{n}(x)\right)$.

No caso divergente os coeficientes satisfazem:

$$
\begin{gathered}
a^{i j} \xi_{i} \xi_{j} \geq \lambda|\xi|^{2} \\
\sum_{i, j=1}^{n} a^{i j}(x) \leq \Lambda^{2} \text { e } \lambda^{-2} \sum_{i, j=1}\left(\left|D_{j} b^{i}(x)\right|^{2}+\left|D_{j} c^{i}(x)\right|^{2}\right)+\lambda^{-1}|d(x)| \leq \gamma^{2}
\end{gathered}
$$

para algum $\Lambda, \lambda>0, x \in \Omega, \xi \in \mathbb{R}^{n}$

Teoremas relativos a desigualdade de Harnack e estimativas de norma Hölder para equações elípticas e parabólicas de segunda ordem desempenham um papel importante na teoria de equações lineares e não lineares. Os primeiros resultados gerais de De Giorgi e Nash a respeito da propriedade de Hölder das soluções foram obtidos no final da década de 50 e deram um poderoso estimulo ao desenvolvimento da teoria das equações elípticas e parabólicas na forma divergente (ver[3-6]).

Comparativamente muito pouco se sabia sobre equações na forma não divergente com coeficientes mensuráveis. Em 1953 no caso bidimensional para equações elípticas Nirenberg obteve estimativas a priori em $c^{1+\alpha} \operatorname{com} \alpha>0$. Em 1956 Corder [8] prova resultados similares para equações elípticas em $\mathbb{R}^{n}$ 
com $n>3$, e obtem estimativas em $c^{\alpha}$ baixo certas restrições sobre a dispersão dos autovalores.

As desigualdades de Harnack e propriedades de Hölder de soluções de equações elípticas e parabólicas foram provadas por E.M. Landis, V. Krylov e M. Safonov (ver[5]).

Esta dissertação está dividida em quatro capítulos.

No capítulo 1, apresentamos a introdução da dissertação.

No capítulo 2, apresentamos resultados preliminares importantes para a compreensão e entendimento dos demais capítulos.

No capítulo 3, estudaremos as propriedades de regularidade do operador $L$ na forma não divergente, desenvolvido a partir do artigo de Safonov ([14]). Veremos regularidade de equações elípticas na forma não divergente. Entre os resultados mais importantes deste capítulo temos os Lemas de Crescimento. Estes lemas levam esse nome pois permitem deduzir a partir de propriedades de uma supersolução $u$ (pode ser $u \geq 1$ ) sobre partes de medidas positivas de uma bola $x_{0}+B_{R / 2}$ (ou uma bola $\left.x_{0}+\varepsilon B_{R / 2}\right)$ propriedades $(u \geq \beta>0$ ) sobre a toda a bola $x_{0}+B_{R / 2}$.

Usando os lemas de crescimento provaremos a desigualdade de Harnack. Podemos interpretar esta desigualdade como segue: se uma solução de uma equação uniformemente elíptica é controlada em um ponto então ela é controlada em qualquer subconjunto compacto do domínio. Provaremos também a propriedade de regularidade de Hölder.

No capítulo 4, estudaremos as propriedades de regularidade do operador $L$ na forma divergente desenvolvido a partir de [15], no qual veremos regularidade de equações elípticas na forma divergente. Entre os resultados mais importantes deste capítulo temos limitações globais de soluções fracas, propriedades locais de soluções fracas, princípio do máximo forte, desigualdade de Harnack e propriedade de regularidade de Hölder. 


\section{2}

\section{Resultados Preliminares}

Neste capítulo apresentaremos alguns resultados necessários para o desenvolvimento e compreensão dos capítulos seguintes.

Teorema 2.1 (Densidade de Lebesgue) Seja $A \subset \mathbb{R}^{n}$ um subconjunto mensurável no sentido de Lebesgue. Define-se a densidade aproximada de A em uma vizinhança $\delta$ de um ponto $x \in \mathbb{R}^{n}$ como $d_{\delta}(x)=\frac{\left|B_{\delta}(x) \cap A\right|}{\mid B_{\delta}(x)}$, onde $B_{\delta}(x)$ denota a bola fechada de raio $\delta$ centrada em $x$. Então tem-se que para quase todo ponto $x \in A, \lim _{\delta \rightarrow 0} d_{\delta}(x)=1$.

\subsection{1}

\section{Espaços $L^{p}$.}

Os resultados desta seção podem ser encontrados em Brezis [1] e em Gilbarg-Trudinger [15].

Definição 2.0.1 Seja $\Omega \subset \mathbb{R}^{n}$ um conjunto aberto e seja $p \in \mathbb{R}$, com $1 \leq p<+\infty$.

Definimos, $L^{p}(\Omega)=\left\{f: \Omega \mapsto \mathbb{R} ; f\right.$ mensuravel e $\left.\int_{\Omega}|f(x)|^{p} d x<+\infty\right\}$.

Então o espaço $L^{p}(\Omega)$, com $1 \leq p<+\infty$,é um espaço de Banach com a seguinte norma,

$$
\|f\|_{L^{p}(\Omega)}=\left(\int_{\Omega}|f(x)|^{p}\right)^{\frac{1}{p}} d x
$$

Definição 2.0.2 Sejam $\Omega \subset \mathbb{R}^{n}$ um conjunto aberto. Definimos $L^{\infty}(\Omega)=\{f: \Omega \mapsto \mathbb{R} ; f$ mensurável e existe $C<0$ tal que $|f(x)| \leq C$ q.s. em $\Omega\}$.

$O$ espaço $L^{\infty}(\Omega)$ é um espaço de Banach com a seguinte norma,

$$
\|f\|_{L^{\infty}(\Omega)}=\inf \{C ;|f(x)| \leq C \text { q.s em } \Omega\} .
$$

Definição 2.0.3 Dizemos que uma função $f: \Omega \mapsto R$ é localmente integrável em $\Omega$, quando $f$ é integrável em todo compacto $K \subset \Omega$. O espaço das funções localmente integráveis é denotado por $L_{l o c}^{1}(\Omega)$. 


\section{Definição 2.0.4}

(i) $C^{k}(\Omega)=\{\phi: \Omega \rightarrow \mathbb{R}$ tal que $\phi$ é $k$ vezes continuamente diferenciável em $\Omega\}$.

(ii) $C^{k}(\bar{\Omega})=\left\{\phi \in C^{k}(\Omega)\right.$ tal que $D^{\alpha} \phi$ é uniformemente continua sobre todos os subconjunto limitado de $\Omega$ para todo $|\alpha| \leq K\}$.

(iii) Denotamos $C_{0}^{\infty}(\Omega)$ o espaço de funções infinitamente diferenciáveis $\phi: U \longrightarrow \mathbb{R}$ com suporte compacto em $\Omega$.

Proposição 2.0.1 $u \in L_{L o c}^{p}(\Omega) \Longleftrightarrow u \in L^{p}(k)$, para todo $k \subset \Omega$ compacto.

Teorema 2.2 (Desigualdade de Holder). Sejam $p, q \in[1,+\infty)$, com $\frac{1}{p}+\frac{1}{q}=1$. Se $f \in L^{p}(\Omega), g \in L^{q}(\Omega)$ então $f g \in L^{1}(\Omega)$ e $\int_{\Omega}|f g| d x \leq\|f\|_{L^{p}(\Omega)}\|g\|_{L^{q}(\Omega)}$.

Teorema 2.3 (Desigualdade de Interpolação) $S e 1 \leq p \leq q \leq r$ então:

(i) $\|u\|_{L^{q}(\Omega)} \leq\|u\|_{L^{p}(\Omega)}^{\lambda}\|u\|_{L^{r}(\Omega)}^{1-\lambda}$ para $u \in L^{r}(\Omega)$, com $\frac{1}{q}=\frac{\lambda}{p}+\frac{(1-\lambda)}{r}$.

(ii) $\|u\|_{L^{q}(\Omega)} \leq \varepsilon\|u\|_{L^{r}(\Omega)}+\varepsilon^{-\mu}\|u\|_{L^{p}(\Omega)}$, onde $\varepsilon>0$ e $\mu=\left(\frac{1}{p}-\frac{1}{q}\right) /\left(\frac{1}{q}-\frac{1}{r}\right)$.

Teorema 2.4 (Desigualdade de Minkowski). Sejam $p \in[1,+\infty)$, e $f, g \in L^{p}(\Omega)$. Então,

$$
\|f+g\|_{L^{p}(\Omega)} \leq\|f\|_{L^{p}(\Omega)}+\|g\|_{L^{p}(\Omega)}
$$

Proposição 2.0.2 Seja $\Omega$ um domínio limitado de $\mathbb{R}^{n}$. Se u é uma função mensurável em $\Omega$ tal que $|u|^{p} \in L^{1}(\Omega)$ para $p \in \mathbb{R}^{n}$, definimos

$$
\Phi_{p}(u)=\left[\frac{1}{|\Omega|} \int_{\Omega}|u|^{p} d x\right]^{\frac{1}{p}} .
$$

Verificam-se as seguintes propriedades:

(i) $\lim _{p \rightarrow \infty} \Phi_{p}(u)=\sup _{\Omega}|u|$.

(ii) $\lim _{p \rightarrow-\infty} \Phi_{p}(u)=\inf _{\Omega}|u|$.

(iii) $\lim _{p \rightarrow 0} \Phi_{p}(u)=\exp \left[\frac{1}{|\Omega|} \int_{\Omega} \log |u| d x\right]$.

Teorema 2.5 (Teorema da Convergência Monótona) Seja $\left(f_{n}\right)$ uma sequência de funções em $L^{1}$ que satisfaz:

(i) $f_{1} \leq f_{2} \leq \ldots \leq f_{n} \leq f_{n+1} \leq \ldots$ 
(ii) $\sup _{n} \int f_{n}<\infty$

Então $f_{n}(x)$ converge q.t.p em $\Omega$ para um limite finito, denotado por $f(x)$, então a função f pertence a $L^{1}$ e $\left\|f_{n}-f\right\|_{1} \rightarrow 0$.

Teorema 2.6 (Teorema da Convergência Dominada) Seja $\left(f_{n}\right)$ uma sequência de funções em $L^{1}$ que satisfaz:

(i) $f_{n}(x) \rightarrow f(x)$ q.t.p em $\Omega$.

(ii) Existe uma função $g \in L^{1}$ tal que para todo $n,\left|f_{n}(x)\right| \leq g(x)$ q.t.p em $\Omega$. Então $f \in L^{1}$ e $\left\|f_{n}-f\right\|_{1} \rightarrow 0$.

Proposição 2.0.3 Sejam $p, q \in[1,+\infty)$, com $p \leq q$ e suponha que $\Omega$ seja um aberto limitado de $R^{n}$. Então $L^{q}(\Omega)$ está contido em $L^{p}(\Omega)$.

Proposição 2.0.4 Sejam $\Omega \subset \mathbb{R}^{n}$ subconjunto aberto e $p \in[1,+\infty)$. Então $C_{0}^{\infty}(\Omega)$ é denso em $L^{p}(\Omega)$.

Teorema 2.7 (Representação de Riesz-Fréchet). Sejam $1 \leq p<+\infty$ e $\varphi \in\left(L^{p}(\Omega)\right)^{\prime}$. Então existe uma única função $u \in L^{q}(\Omega) \operatorname{com} \frac{1}{p}+\frac{1}{q}=1$, tal que,

$$
<\varphi, f>_{\left.\left(L^{p}(\Omega)\right)^{\prime} \times L^{p}(\Omega)\right)}=\int_{\Omega} u f d x
$$

para todo $f \in L^{p}(\Omega)$. Além disso, $\|u\|_{\left(L^{p}(\Omega)\right)}=\|\varphi\|_{\left(\left(L^{p}(\Omega)\right)^{\prime}\right)}$.

\subsection{2}

\section{Espaços de Sobolev}

Os resultados desta seção podem ser encontrados em Evans[2], Brezis[1] e Gilbarg-Trudinger [15].

Definição 2.0.5 Seja $u \in L_{L o c}^{1}$ suponhamos que existe $v \in L_{L o c}^{1}(\Omega)$ tal que $\forall \phi \in C_{0}^{\infty}(\Omega)$ temos,

$$
\int_{\Omega} u \frac{\partial \phi}{\partial x_{i}}=-\int_{\Omega} v \phi
$$

Chamamos $v$ de derivada fraca em $x_{i}$ de $u$ e escrevemos $v=\frac{\partial u}{\partial x_{i}}$.

Definição 2.0.6 Se $\int u \frac{\partial^{k} \phi}{\partial x_{1}^{\alpha} \ldots \partial x_{n}^{\alpha}}=(-1)^{|\alpha|} \int \omega \phi$, para todo $\phi \in C^{\infty}(\Omega)$, $|\alpha|=\alpha_{1}+\alpha_{2}+\ldots+\alpha_{n}$, então $\omega$ é por definição a derivada fraca $\frac{\partial^{k} u}{\partial x_{1}^{\alpha_{1}} \ldots \partial x_{n}^{\alpha_{n}}}$.

Proposição 2.0.5 (Unicidade da Derivada Fraca) Se existe $v_{1}, v_{2}$ tal que

$$
-\int v_{2} \phi=\int u \frac{\partial \phi}{\partial x_{1}}=\int v_{1} \phi
$$

para todo $\phi \in C_{0}^{\infty}(\Omega)$, então $v_{1}=v_{2}$ q.t.p. 
Lema 2.0.1 (Du Bois Raymond). Seja $u \in L_{\text {loc }}^{1}(\Omega)$. Então $\int_{\Omega} u \phi=0$ para todo $\phi \in C_{0}^{\infty}(\Omega)$ se, e somente se, $u=0$ q.t.p em $\Omega$.

Definição 2.0.7 Define-se o espaço de Sobolev

$W^{k, p}(U)=\left\{u \in L_{\text {loc }}^{1}(U) \mid\right.$ para todo $|\alpha| \leq k, D^{\alpha} u$ existe no sentido fraco e $\left.D^{\alpha} u \in L^{p}(U)\right\}$

Observação 2.0.1 Se $p=2$, escrevemos $H^{k}(U)=W^{k, 2}(U)$ com $k=0,1, \ldots$ Observe que $H^{0}(U)=L^{2}(U)$.

Definição 2.0.8 Se $u \in W^{k, p}(U)$, definimos a norma

$$
\|u\|_{W^{k, p}}:=\left\{\begin{array}{cc}
\left(\sum_{|\alpha| \leq k} \int_{U}\left|D^{\alpha} u\right|^{p} d x\right)^{\frac{1}{p}} & (1 \leq p \leq \infty) \\
\left(\sum_{|\alpha| \leq k} \text { supess }_{U}\left|D^{\alpha} u\right|\right. & (p=\infty)
\end{array}\right.
$$

Teorema $2.8 W^{k, p}(U)$ é um espaço de Banach.

Teorema 2.9 $H^{k}(U)$ é um espaço de Hilbert.

Teorema 2.10 (Friedrichs) Seja $u \in W^{1, p}(\Omega)$ com $1 \leq p<\infty$.Então existe uma sequência $\left(u_{n}\right)$ em $C_{0}^{\infty}\left(R^{N}\right)$ tal que:

(i) $u_{n \mid \Omega} \rightarrow$ e em $L^{p}(\Omega)$,

(ii) $\nabla u_{n \mid \omega} \rightarrow \nabla u_{\mid \omega}$ em $L^{p}(\omega)^{N}$ para todo $\omega \subset \subset \Omega$. Quando $\Omega=R^{N} e$ $u \in W^{1, p}\left(R^{N}\right)$ com $1 \leq p<\infty$, existe uma sequência $\left(u_{n}\right)$ em $C_{0}^{\infty}\left(R^{N}\right)$ tal que,

$$
\begin{gathered}
u_{n} \rightarrow u e m L^{p}\left(R^{N}\right), \\
\nabla u_{n} \rightarrow \nabla u e m L^{p}\left(R^{N}\right)^{N}
\end{gathered}
$$

Proposição 2.0.6 (Diferenciação de um Produto)

Sejam $u, v \in W^{1, p}(\Omega) \cap L^{\infty}(\Omega)$ com $1 \leq p \leq \infty$. Então $u v \in W^{1, p}(\Omega) \cap L^{\infty}(\Omega)$ $e$

$$
\frac{\partial}{\partial x_{i}}(u v)=\frac{\partial u}{\partial x_{i}} v+u \frac{\partial v}{\partial x^{i}}
$$

com $i=1,2, \ldots, N$.

Proposição 2.0.7 (Diferenciação de uma Composição) Seja $G \in C^{1}(R)$ tal que $G(0)=0$ e $\left|G^{\prime}(s)\right| \leq M$, para todo $s \in R$ para alguma constante $M$. Seja $u \in W^{1, p}(\Omega) \operatorname{com} 1 \leq p \leq \infty$. Então,

$$
G \circ u \in W^{1, p}(\Omega)
$$


$e$

$$
\frac{\partial}{\partial x_{i}}(G \circ u)=\left(G^{\prime} \circ u\right) \frac{\partial u}{\partial x_{i}}
$$

com $i=1,2, \ldots, N$.

Definição 2.0.9 Um domínio limitado $\Omega \subset \mathbb{R}^{n}$ tem fronteira de classe $C^{k, \alpha}$ com $0<\alpha \leq 1$ se em cada ponto $X_{0}$ pertencente à fronteira de $\Omega$ existe uma bola $B=B\left(x_{0}\right)$ e uma aplicação injetora $\Psi$ de $B$ sobre $D \subset \mathbb{R}^{n}$ que satisfaz:

(i) $\Psi(B \cap \Omega) \subset \mathbb{R}_{+}^{n}$,

(ii) $\Psi(B \cap \partial \Omega) \subset \partial \mathbb{R}_{+}^{n}$,

(iii) $\Psi \in C^{k, \alpha}(B), \Psi^{-1} \in C^{k, \alpha}(D)$.

Teorema 2.11 (Imersões de Sobolev) Seja $\Omega$ um $C^{0,1}$ domínio em $\mathbb{R}^{n}$. Tem-se as seguintes imersões:

(i) Se $m p<n$, então $W^{m, p}(\Omega)$ é imerso continuamente em $L^{p^{*}}(\Omega)$, em que $p^{*}=\frac{n p}{n-m p}$ e imerso compactamente em $L^{q}(\Omega)$, para todo $1 \leq q<\frac{n p}{n-m p}=$ $p^{*}$.

(ii) Se $0 \leq k<m-\frac{n}{p}<k+1$ o espaço $W^{m, p}(\Omega)$ é continuamente imerso em $C^{k, \alpha}(\bar{\Omega}), \alpha=m-\frac{n}{p}-k$ e compactamente imerso em $C^{k, \beta}(\bar{\Omega})$ para todo $\beta<\alpha$.

Definição 2.0.10 Seja $\Omega$ um subconjunto aberto de $\mathbb{R}^{N}$. Definimos $W_{0}^{m, p}(\Omega)={\overline{C_{0}^{\infty}}(\Omega)}^{W^{m, p}(\Omega)}$.

No caso $p=2$, o espaço $W_{0}^{m, p}(\Omega)$ será representado por $H_{0}^{m}(\Omega)$.

Teorema 2.12 (Desigualdade de Poincaré). Sejam $\Omega \subset \mathbb{R}^{N}$ uma aberto limitado e $1 \leq p<+\infty$. Então existe uma constante $C$ (dependendo de $\Omega$ e p), tal que

$$
\|u\|_{L^{p}(\Omega)} \leq C\|\nabla u\|_{L^{p}(\Omega)}
$$

para todo u em $W_{0}^{1, p}(\Omega)$.

Teorema 2.13 Seja $\Omega$ aberto, limitado de $\mathbb{R}^{n}$ de classe $C^{m}$, então $C^{m}(\bar{\Omega})$ é denso em $W^{m, p}(\Omega)$ para todo $1 \leq p<\infty$.

Teorema 2.14 Seja $f$ uma função diferenciável por partes em $\mathbb{R}$ com $f^{\prime} \in$ $L^{\infty}(\mathbb{R}), f(0)=0$. Então se $u \in W^{1, p}(\Omega)$, temos $f \circ u \in W^{1, p}(\Omega)$. Além disso, denotando por $L$ o conjunto do pontos onde $f$ não é diferenciável por partes, temos

$$
D(f \circ u)=\left\{\begin{array}{cl}
f^{\prime}(u) D u & \text { se } u \notin L \\
0 & \text { se } u \in L .
\end{array}\right.
$$


Teorema 2.15 Seja $u \in W^{1,2}(\Omega)$, onde $\Omega$ é convexo e suponha que existe uma constante $k$ tal que, para todo $B_{R} \subset \Omega$,

$$
\int_{\Omega \cap B_{R}}|D u|^{2} d x \leq k R^{n-1}
$$

Então existem constantes positivas $\sigma_{0}$ e $C=C(n)$ tais que,

$$
\int_{\Omega} e^{\frac{\sigma}{k}\left|u-u_{\Omega}\right|} d x \leq C(\operatorname{diam} \Omega)^{n}
$$

em que $\sigma=\sigma_{0}(\Omega)(\operatorname{diam} \Omega)^{-n}$. 


\section{Regularidades de Equações Elípticas na Forma Não Diver- gente}

Neste capítulo abordaremos regularidade de equações elípticas na forma não divergente. Os resultados deste capítulo foram obtidos seguindo as ideias do famoso artigo de Safonov (ver [14]) em que ele dá uma demonstração relativamente simples e baseada somente na desigualdade ABP (veja Teorema 3.1 abaixo) dos resultados fundamentais que obteve com Krylov.

Consideremos o operador elíptico

$$
L=a^{i j}(x) \frac{\partial^{2}}{\partial x^{i} \partial x^{j}}+B^{i}(x) \frac{\partial}{\partial x_{i}}-c(x),
$$

com coeficientes mensuráveis e limitados. Em particular, suponhamos que existe $0<\lambda<1$ tal que para todo $\xi=\left(\xi^{1}, \ldots, \xi^{d}\right) \in \mathbb{R}^{n}, x \in \mathbb{R}^{n}$ temos,

$$
\lambda|\xi|^{2} \leq a^{i j}(x) \xi^{i} \xi^{j} \leq \lambda^{-1}|\xi|^{2},|B(x)| \leq \lambda^{-1}, 0 \leq c(x) \leq \lambda^{-1},
$$

em que $B(x)=\left(B^{1}(x), \ldots, B^{d}(x)\right)$.

Nesse capítulo trabalharemos com o espaço de Sobolev $W^{2, n}(\Omega)$ definido por,

$$
W^{2, n}(\Omega)=\left\{f: \Omega \subset \mathbb{R}^{n} \mapsto \mathbb{R} / D^{\alpha} f \in L^{n}(\Omega), \alpha \in \mathbb{N}^{n}, \alpha_{1}+\cdots+\alpha_{n} \leq 2\right\}
$$

O seguinte teorema fundamental é conhecido como desigualdade ABP, de Alexandrov-Bakelman-Pucci. Ele é uma estimativa a priori para o tamanho de uma solução $u$ do problema de Dirichlet em termos do tamanho de $L u$.

Teorema 3.1 Seja o domínio

$\Omega \subseteq B_{R}=\left\{x \in \mathbb{R}^{n}:|x|<R\right\}, 0<R \leq 1$ e uma função $u \in W^{2, n}(\Omega) \cap C(\bar{\Omega})$ com $u=0$ em $\partial \Omega$ e $u>0$ sobre $\Omega$. Então temos a estimativa

$$
\max _{\bar{\Omega}}\{u\} \leq A R\left|(L u)_{-}\right|_{L^{n}(\Omega)}
$$

em que a constante $A$ depende apenas de $n$ e $\lambda$ e $(L u)_{-}=\max \{-L u, 0\}$.

Demonstração. Seja o conjunto de contato dos pontos em que o gráfico de $u$ pode ser tocado por cima por um hiperplano,

$$
\Gamma^{+}=\left\{y \in \Omega / \exists p \in \mathbb{R}^{n}: u(x) \leq u(y)+p(x-y), \text { para todo } x \in \Omega\right\}
$$


Podemos verificar que $u$ é uma função côncava em $\Omega$ se, e somente se $\Gamma^{+}=\Omega$. Também quando $u \in C^{1}(\Omega)$ tem-se que $p=D u(y)$, além do mais quando $u \in C^{2}(\Omega)$ a matriz Hessiana $D^{2} u=D_{i j} u$ é não positiva em $\Gamma^{+}$. Em geral $\Gamma^{+}$ é fechado relativo a $\Omega$, para todo $u \in C(\Omega)$.

Para $y \in \Omega$ define-se o mapa normal,

$$
\chi(y)=\chi_{u}(y)=\left\{p \in \mathbb{R}^{n} / u(x) \leq u(y)+p(x-y), \forall x \in \Omega\right\}
$$

Se $A \subset \Omega$, definimos $\chi(A)=\cup_{y \in A} \chi(y)$.

Temos que $\chi(y) \neq \emptyset$ se e, somente se, $y \in \Gamma^{+}$e quando $u \in C^{1}(\Omega)$, $\chi(y)=D u(y)$ em $\Gamma^{+}$.

Como um exemplo de uma função não diferenciável " $u$ ", seja $\Omega$ a bola $B=B_{R}(z)$ e $u$ a função cujo gráfico é um cone com base $\Omega$ e vértice $(z, a)$ para algum $a \in \mathbb{R}$, isto é, $u(x)=a\left(1-\frac{|x-z|}{R}\right)$.

Então tem-se,

$$
\chi(y)=\left\{\begin{array}{lll}
\frac{-a(y-z)}{R|y-z|} & \text { para } & y \neq z \\
B_{a / R}(0) & \text { se } & y=z,
\end{array}\right.
$$

pois

$$
\begin{aligned}
\chi(z) & =\left\{p \in \mathbb{R}^{n} / u(x) \leq u(z)+p \cdot(x-z) \forall x \in \Omega\right\} \\
& =\left\{p \in \mathbb{R}^{n} / a\left(1-\frac{|x-z|}{R}\right) \leq a+p \cdot(x-z) \forall x \in \Omega\right\} \\
& =\left\{p \in \mathbb{R}^{n} /-\frac{a}{R} \leq p \cdot \frac{x-z}{|x-z|} \forall x \in \Omega\right\} \\
& =\left\{p \in \mathbb{R}^{n} / \frac{p \cdot(z-x)}{|z-x|} \leq \frac{a}{R} \forall x \in \Omega\right\} \\
& =\left\{p \in B_{a / R}(0)\right\} .
\end{aligned}
$$

Lema 3.3.1 Para $u \in C^{2}(\Omega) \cap C^{0}(\bar{\Omega})$ tem-se

$$
\sup _{\Omega} u \leq \frac{n}{w_{n}^{1 / n}}\left(\int_{\Gamma^{+}}\left|\operatorname{det} D^{2} u\right|\right)^{1 / n}
$$

em que $\omega_{n}$ é o volume da bola unitária em $\mathbb{R}^{n}$.

Demonstração. Denotamos por $|\chi(\Omega)|$ a $n$-dimensional medida de Lebesgue de $\chi(\Omega)$. Assim, temos 


$$
\begin{aligned}
|\chi(\Omega)| & =\left|\chi\left(\Gamma^{+}\right)\right|=\left|D u\left(\Gamma^{+}\right)\right| \\
& =\int_{D u\left(\Gamma^{+}\right)} 1 d z, \text { fazendo } \mathrm{z}=\mathrm{Du} \text { e } \mathrm{dz}=\operatorname{det}(\mathrm{D}(\mathrm{Du})) \\
& =\int_{\Gamma^{+}} \operatorname{det}|D(D u)| \\
& =\int_{\Gamma^{+}} \operatorname{det}\left(D^{2} u\right) \\
& \leq \int_{\Gamma^{+}}\left|\operatorname{det} D^{2} u\right|
\end{aligned}
$$

Agora provaremos que $u$ pode ser estimado em termos de $|\chi(\Omega)|$. Suponha que $u$ tome um máximo positivo num ponto $y \in \Omega$ e seja $k$ a função cujo gráfico é o cone $K$ com vértice $(y, u(y))$ e base uma bola com raio 1. Pela hipótese do teorema esta bola contém $\Omega$ e $\operatorname{logo} \chi_{k}(\Omega) \subset \chi_{u}(\Omega)$, desde que para cada hiperplano que toca o gráfico de $k$ por cima, existe um hiperplano paralelo tangente ao gráfico de $u$ em um ponto interior de $\Omega$. Logo toda inclinação em $\chi_{k}(\Omega)$ também é uma inclinação de $\chi_{u}(\Omega)$.

$\operatorname{Assim} \chi_{k}(\Omega) \subset \chi_{k}(\Omega)$ e consequentemente

$$
\left|\chi_{\tilde{k}}(\Omega)\right| \leq\left|\chi_{u}(\Omega)\right|
$$

logo de (3.4) e (3.5) tem-se

$$
w_{n}(u(y))^{n} \leq \int_{\Gamma^{+}}\left|\operatorname{det} D^{2} u\right|
$$

e logo,

$$
u(y) \leq \frac{1}{w_{n}^{1 / n}}\left(\int_{\Gamma^{+}}\left|\operatorname{det} D^{2} u\right|\right)^{1 / n}
$$

e $\log o$

$$
\sup _{\Omega} u \leq \frac{1}{w_{n}^{1 / n}}\left(\int_{\Gamma^{+}}\left|\operatorname{det} D^{2} u\right|\right)^{1 / n}
$$

O caso especial do Teorema 3.1 quando $b=0$ segue do Lema 3.3.2 através da desigualdade matricial $\operatorname{det}(A) \operatorname{det}(B) \leq\left(\frac{\operatorname{tr}(A B)}{n}\right)^{n}$, onde $A$ e $B$ são matrizes simétricas e $A, B \geq 0$.

Com efeito, considerando $A=-D^{2} u, B=a^{i j}$ temos sobre $\Gamma^{+}$

$$
\begin{aligned}
\left|\operatorname{det} D^{2} u\right| & =\operatorname{det}\left(-D^{2} u\right) \\
& \leq \frac{1}{\operatorname{det}\left(a^{i j}\right)}\left(\frac{-a^{i j} D_{i j} u}{n}\right)^{n}
\end{aligned}
$$


logo do Lema 3.3.1 tem-se que

$$
\begin{aligned}
\sup _{\Omega} u & \leq \frac{1}{w_{n}^{\frac{1}{n}}}\left(\int_{\Gamma^{+}}\left|\operatorname{det} D^{2} u\right|\right)^{\frac{1}{n}} \\
& \leq \frac{1}{w_{n}^{\frac{1}{n}}}\left(\int_{\Gamma^{+}}\left[\frac{1}{D}\left(-\frac{a^{i j} D_{i j} u}{n}\right)^{n}\right]\right)^{\frac{1}{n}} \\
& =\frac{1}{w_{n}^{\frac{1}{n}} n}\left[\int_{\Gamma+}\left(\frac{\left(-a^{i j} D_{i j} u\right)}{\left(\operatorname{det} a^{i j}\right)^{\frac{1}{n}}}\right)^{n}\right]^{\frac{1}{n}} \\
& \leq \frac{1}{w_{n}^{\frac{1}{n}} n}\left|\frac{a^{i j} D_{i j} u}{\left(\operatorname{det} a^{i j}\right)^{\frac{1}{n}}}\right|_{L^{n}\left(\Gamma^{+}\right)}
\end{aligned}
$$

De onde obtemos para $b=0$ o seguinte lema.

Lema 3.3.2 Para $u \in C^{2}(\Omega) \cap C(\bar{\Omega})$ tem-se

$$
\sup _{\Omega} u \leq \frac{1}{d w_{n}^{1 / n}}\left|\frac{-a^{i j} D_{i j} u}{\operatorname{det}\left(a^{i j}\right)^{1 / n}}\right|_{L^{n}\left(\Gamma^{+}\right)}
$$

Para tratar o caso geral para $b \neq 0$, vamos mostrar o seguinte lema.

Lema 3.3.3 Seja $g \geq 0$ uma função localmente integrável em $\mathbb{R}^{n}$. Então para qualquer $u \in C^{2}(\Omega) \cap C(\bar{\Omega})$ tem-se

$$
\begin{aligned}
\int_{B_{\tilde{M}}(0)} & g \leq \int_{\Gamma^{+}} g(D u)\left|\operatorname{det} D^{2} u\right| \\
\leq & \int_{\Gamma^{+}} g(D u)\left(\frac{-a^{i j} D_{i j} u}{n\left(\operatorname{det}\left(a^{i j}\right)\right)^{1 / n}}\right)^{n}
\end{aligned}
$$

em que $\tilde{M}=\sup _{\Omega} u$.

Demonstração. Temos pelo Teorema de mudança de variáveis em integrais

$$
\int_{\chi_{u}(\Omega)} g=\int_{\chi_{u}\left(\Gamma^{+}\right)} g=\int_{\Gamma^{+}} g(D u)\left|\operatorname{det} D^{2} u\right|
$$

e como $\chi_{\tilde{k}}(\Omega) \subset \chi_{u}(\Omega)$ então de (3.4), (3.6) e (3.7), tem-se

$$
\begin{aligned}
\int_{B_{\tilde{M}}(0)} g & \leq \int_{\chi_{\tilde{k}}(\Omega)} g \leq \int_{\Gamma^{+}} g(D u)\left|\operatorname{det} D^{2} u\right| \\
& \leq \int_{\Gamma^{+}} g(D u)\left(\frac{-a^{i j} D_{i j} u}{n\left(\operatorname{det}\left(a^{i j}\right)\right)^{1 / n}}\right)^{n} .
\end{aligned}
$$

Agora consideremos

$$
g(p)=\left(|p|^{\frac{n}{n-1}}+\gamma^{\frac{n}{n-1}}\right)^{1-n} \text { para } \gamma>0 \text { a fixar posteriormente. }
$$


Usando a desigualdade de Hölder tem-se em $\Omega^{+}=\{x \in \Omega / u(x)>0\}$

$$
\begin{aligned}
\frac{-a^{i j} D_{i j} u}{n\left(\operatorname{det}\left(a^{i j}\right)\right)^{1 / n}} \leq \frac{B^{i} D_{i} u-L u}{n\left(\operatorname{det}\left(a^{i j}\right)\right)^{1 / n}} \leq B^{i} D_{i} u+(L u)^{-}\left(\text {pois }-L u \leq(L u)^{-}\right) \\
\quad \leq \frac{|B||D u|+\left|(L u)^{-}\right|}{n\left(\operatorname{det}\left(a^{i j}\right)\right)^{1 / n}} \\
\leq \frac{\left(|B||D u|+\gamma^{-1}|L u| \gamma\right)}{n\left(\operatorname{det}\left(a^{i j}\right)\right)^{1 / n}}\left(\frac{1}{n}+\frac{1}{\frac{n}{n-1}}=1\right) \\
\leq \frac{\left(|B|^{d}+\gamma^{-n}|L u|^{n}\right)^{1 / n}}{n\left(\operatorname{det}\left(a^{i j}\right)\right)^{1 / n}}(\underbrace{|D u|^{\frac{n}{n-1}}+\gamma^{\frac{n}{n-1}}}_{(g(D u))^{-1 / n}})^{\frac{n-1}{n}}
\end{aligned}
$$

Logo do Lema 3.3.3 e (3.8) tem-se

Como

$$
\begin{aligned}
\int_{B_{\tilde{M}}(0)} g & \leq \int_{\Gamma^{+}} g(D u) \cdot\left(g(D u)^{-1 / n}\right)^{n}\left(\frac{|B|^{n}+\gamma^{-n}(L u)^{n}}{n^{n}\left(\operatorname{det}\left(a^{i j}\right)\right)}\right) \\
& =\frac{1}{n^{n}} \int_{\Gamma^{+}} \frac{|B|^{n}+\gamma^{-n}|L u|^{n}}{\operatorname{det}\left(a^{i j}\right)} .
\end{aligned}
$$

$$
g(p) \geq 2^{2-n}\left(|p|^{n}+\gamma^{n}\right)^{-1},
$$

temos

$$
\begin{aligned}
\int_{B_{\tilde{M}}(0)} g(p) & \geq \int_{B_{\tilde{M}}(0)} 2^{2-n}\left(|p|^{n}+\gamma^{d}\right)^{-1} \\
& =\log \left(\frac{\tilde{M}^{n}}{\gamma^{n}}+1\right) w_{n} \cdot 2^{2-n}
\end{aligned}
$$

Assim de (3.9) e (3.10)

$$
\log \left(\frac{\tilde{M}^{n}}{\gamma^{n}}+1\right) \leq \frac{2^{n-2}}{w_{n} n^{n}} \int_{\Gamma^{+}} \frac{|B|^{n}+\gamma^{-n}|L u|^{n}}{\operatorname{det}\left(a^{i j}\right)}=I
$$

Se $\gamma=\left|\frac{(L u)^{-}}{\left(\operatorname{det}\left(a^{i j}\right)\right)^{1 / n}}\right|_{L^{n}\left(\Gamma^{+}\right)}$, então

$$
I \leq \frac{2^{n-2}}{w_{n} n^{n}} \int_{\Gamma^{+}}\left(\frac{|B|^{n}}{\operatorname{det}\left(a^{i j}\right)}+1\right) .
$$

Assim de (3.11) e (3.12)

$$
\begin{aligned}
\tilde{M} & \leq\left(\exp \left(\frac{2^{n-2}}{w_{n} n^{n}} \int_{\Gamma^{+}} \frac{|B|^{n}}{\operatorname{det}\left(a^{i j}\right)}+1\right)-1\right)^{1 / n}\left|\frac{(L u)^{-}}{\operatorname{det}\left(a^{i j}\right) n}\right|_{L^{n}\left(\Gamma^{+}\right)} \\
& \leq A R\left|(L u)^{-}\right|_{L^{n}\left(\Gamma^{+}\right)} \leq A R\left|(L u)^{-}\right|_{L^{n}(D)}
\end{aligned}
$$

em que $A=A(n, \gamma)$.

Assim,

$$
\sup _{\bar{\Omega}} u \leq A R\left|(L u)^{-}\right|_{L^{n}(\Omega)},
$$

ficando provado o Teorema 3.1 quando $u \in C^{2}(\Omega) \cap C(\bar{\Omega})$. 
Se $u \in W^{2, n}(\Omega) \cap C(\bar{\Omega})$, como $C^{2}(\bar{\Omega})$ é denso em $W^{2, n}(\Omega)$ existe $\left(u_{m}\right) \subset C^{2}(\Omega) \cap C(\bar{\Omega})$ tal que $u_{m} \rightarrow u$ em $W^{2, n}(\Omega)$.

Tem-se,

$$
\left|\left(L u_{m}\right)^{-}\right|_{L^{n}(\Omega)} \leq\left|\left(L\left(u_{m}-u\right)\right)^{-}\right|_{L^{n}(\Omega)}+\left|(L u)^{-}\right|_{L^{n}(\Omega)}
$$

Também se $u_{m} \rightarrow u$ em $W^{2, n}(\Omega) \Rightarrow u_{m} \rightarrow u$ em $C(\bar{\Omega})$, logo

$$
\sup _{\Omega} u_{m} \rightarrow \sup _{\Omega} u
$$

Assim de (3.13) aplicado a $u_{m}$ tem-se

$$
\sup _{\bar{\Omega}} u_{m} \leq A R\left|\left(L u_{m}\right)^{-}\right|_{L^{n}(\Omega)} .
$$

Logo de (3.14), (3.15), usando o Teorema da convergência dominada de Lebesgue,

$$
\sup _{\bar{\Omega}} u \leq A R\left|(L u)^{-}\right|_{L^{n}(\Omega)},
$$

ficando provado o Teorema 3.1.

A partir do Teorema 3.1, mediante um raciocínio por absurdo podemos provar o Principío do Máximo para funções em $W^{2, n}(\Omega)$.

Corolário 3.2 (Principio do Máximo para funções em $W^{2, n}(\Omega)$ )

Se $u_{1}, u_{2} \in W^{2, n}(\Omega) \cap C(\bar{\Omega})$, com $u_{1} \geq u_{2}$ em $\partial \Omega, L u_{1} \leq L u_{2}$ (q.s em $\Omega$ ) então $u_{1} \geq u_{2}$ em $\Omega$.

Demonstração. Por absurdo

Seja $u=u_{2}-u_{1}$, e $\Omega_{1}$ é uma componente conexa não vazia de $\{u>0\}$ arbitraria. Como $u \leq 0$ em $\partial \Omega$ e $u$ é continua, temos $u=0$ em $\partial \Omega_{1}$. Assim pelo Teorema 3.1 aplicando a $\Omega_{1}$, tem-se

$$
\begin{gathered}
\max _{\overline{\Omega_{1}}}\{u\} \leq A R\left|(L u)_{-}\right|_{L^{n}(\Omega)}, \\
\max _{\overline{\Omega_{1}}}(L u)_{-}=\max \{-L u, 0\}=0 \\
\Rightarrow \max _{\overline{\Omega_{1}}}\{u\} \leq A R|0|_{L_{n}(\Omega)}=0
\end{gathered}
$$

Absurdo, pois $\Omega_{1}$ é não vazio.

Do Princípio do Máximo podemos ver que se uma função é maior ou igual a zero na fronteira e o operador aplicado a $L$ é menor ou igual a zero, então a função é maior ou igual a zero em todo o domínio.

$\mathrm{Na}$ continuação vamos precisar de algumas definições. 
Seja $R_{0}, 0<\mu_{0}<1$ e um conjunto $\Gamma \subseteq B_{R_{0}}$, com $|\Gamma|>0$. Com respeito a $\Gamma, R_{0}, \mu_{0}$ construiremos um subconjunto aberto $\Gamma_{0} \subseteq B_{R_{0}}$.

Denote por $\vartheta$ o sistema de bolas $B=x+B_{R_{0}}$ tal que $B \subseteq B_{R_{0}},|B \cap \Gamma| \geq$ $\mu_{0}|B|$. Então definimos $\Gamma_{0}$ :

$$
\Gamma_{0}=\cup \vartheta \subseteq B_{R_{0}}
$$

Observamos que:

(i) $\Gamma_{0}$ é aberto pois é uma união de abertos $B$ de $\vartheta$.

(ii) Se $|\Gamma| \geq \mu_{0}\left|B_{R_{0}}\right|$ então $\Gamma_{0}=B_{R_{0}}$. Com efeito, neste caso $B_{R_{0}} \subset \vartheta$ pois,

$$
\left|\Gamma \cap B_{R_{0}}\right|=|\Gamma| \geq \mu_{0}\left|B_{R_{0}}\right|
$$

Assim,

$$
B_{R_{0}} \subset \cup_{B \subset \vartheta} B=\Gamma_{0},
$$

$\log \mathrm{O}$

$$
\Gamma_{0}=B_{R_{0}}
$$

Na continuação provaremos um resultado técnico da Teoria de Medida, usando o conceito de ponto de Densidade de Lebesgue, e que será de suma importância na prova dos Lemas de Crescimento.

Lema 3.3.4 $\left|\Gamma \backslash \Gamma_{0}\right|=0$ e se $|\Gamma|<\mu_{0}\left|B_{R_{0}}\right|$, então $\left|\Gamma_{0}\right| \geq\left(1+\frac{1-\mu_{0}}{3^{n}}\right)|\Gamma|$.

Demonstração. Suponha $R_{0}=1$. Vamos demonstrar que $\left|\Gamma \subset \Gamma_{0}\right|=0$.

Como $|\Gamma|>0$, quase todo $x \in \Gamma$ é um ponto de densidade, i.e. $\lim _{\delta \rightarrow 0} \frac{\left|B_{\delta}(x) \cap \Gamma\right|}{\left|B_{\delta}(x)\right|}=1$.

Observe que $B_{\delta}(x) \cap \Gamma \subset B_{\delta}(x)$, então

$$
\left|B_{\delta}(x) \cap \Gamma\right| \leq\left|B_{\delta}(x)\right| \text {, dai temos } \frac{\left|B_{\delta}(x) \cap \Gamma\right|}{\left|B_{\delta}(x)\right|} \leq 1
$$

Como $\lim _{\delta \rightarrow 0} \frac{\left|B_{\delta}(x) \cap \Gamma\right|}{\left|B_{\delta}(x)\right|}=1$, dado $\varepsilon>0$ existe $\delta_{0}>0$ tal que $\left|\frac{B_{\delta}(x) \cap \Gamma}{B_{\delta}(x)}-1\right| \leq \varepsilon$ para todo $0<|\delta| \leq \delta_{0}$ de onde para $\delta=\delta_{0}$ tem-se,

$$
1-\varepsilon \leq \frac{\left|B_{\delta_{0}}(x) \cap \Gamma\right|}{\left|B_{\delta_{0}}(x)\right|} \leq 1+\varepsilon
$$

Considere $\varepsilon=1-\mu_{0}$, logo temos que existe $\delta_{0}>0$ tal que

$$
\mu_{0} \leq \frac{\left|B_{\delta_{0}}(x) \cap \Gamma\right|}{\left|B_{\delta_{0}}(x)\right|} \Rightarrow\left|B_{\delta_{0}}(x) \cap \Gamma\right| \geq \mu_{0}\left|B_{\delta_{0}}(x)\right|,
$$

então, $x \in \Gamma_{0}$. Por tanto $\left|\Gamma \backslash \Gamma_{0}\right|=0$. 
Seja $\Gamma \subset B,|\Gamma|<\mu_{0}\left|B_{0}\right|$.

Define-se,

$$
\vartheta^{0}=\left\{B \in \vartheta:|B \cap \Gamma|=\mu_{0}|B|\right\} .
$$

Seja $B=x+B_{R} \in \vartheta, 0 \leq \theta \leq 1$, $B^{\theta}=(1-\theta) x+B_{R}(0)+\theta(1-R)=B_{R+\theta(1-R)}((1-\theta) x)$.

Definimos a seguinte função,

$$
\begin{aligned}
\rho:[0,1] & \mapsto[0,1] \\
\theta & \rightarrow \rho(\theta)=\frac{\left|B^{\theta} \cap \Gamma\right|}{\left|B^{\theta}\right|}
\end{aligned}
$$

Afirmação: $\rho$ é continua, $\rho(0) \geq \mu_{0}$ e $\rho(1)<\mu_{0}$.

Provemos que $\rho(0) \geq \mu_{0}$.

Com efeito, como $\rho(0)=\frac{\left|B^{0} \cap \Gamma\right|}{\left|B^{0}\right|}, B^{0}=x+B_{R} \in \vartheta$, temos

$$
\left|B^{0} \cap \Gamma\right| \geq \mu_{0}\left|B^{0}\right|
$$

Assim,

$$
\rho(0)=\frac{\left|B^{0} \cap \Gamma\right|}{\left|B^{0}\right|} \geq \mu_{0} .
$$

Provemos que $\rho(1)<\mu_{0}$.

Dado que

$$
\begin{aligned}
\rho(1) & =\frac{\left|B^{\prime} \cap \Gamma\right|}{\left|B^{\prime}\right|}, B^{\prime}=0+B_{1}=B_{1} \text { e }\left|B_{1} \cap \Gamma\right|=|\Gamma|<\mu_{0}\left|B_{1}\right|, \\
& \text { deduzimos } \rho(1)=\frac{\left|B^{\prime} \cap \Gamma\right|}{\left|B_{1}\right|}<\mu_{0} . \\
& \text { Provemos que } \rho \text { é continua. }
\end{aligned}
$$

Se $\theta_{n} \rightarrow \theta$ então

$$
\left|B^{\theta^{n}}\right|=\left|B_{R-\theta^{n}(1-R)}\left(\left(1-\theta^{n}\right) x\right)\right| \rightarrow\left|B_{R+\theta(1-R)}((1-\theta) x)\right|=\left|B^{\theta}\right| .
$$

Análogo,

$$
\left|B^{\theta^{n}} \cap \Gamma\right| \rightarrow\left|B^{\theta} \cap \Gamma\right|
$$

$\mathrm{e}$

$$
\rho\left(\theta^{n}\right)=\frac{\left|B^{\theta^{n}} \cap \Gamma\right|}{\left|B^{\theta^{n}}\right|} \rightarrow \frac{\left|B^{\theta} \cap \Gamma\right|}{\left|B^{\theta}\right|}=\rho(\theta) .
$$

Assim $\rho:[0,1] \mapsto[0,1]$ é continua.

Logo para algum $\theta_{0}, \rho\left(\theta_{0}\right)=\mu_{0}$ (pelo Teorema do Valor Intermediário). Logo $B^{\theta_{0}} \in \vartheta^{0}$ e também

$$
\begin{gathered}
B=x+B_{R}, B^{\theta_{0}}=\left(1-\theta_{0}\right) x+B_{R+\theta_{0}(1-R)}(0), \\
B \subset B^{\theta_{0}} .
\end{gathered}
$$


Se $y \in B,|y-x| \leq R$, temos

$$
\begin{aligned}
\left|y-\left(1-\theta_{0}\right) x\right|= & \left|y-x+\theta_{0} x\right| \leq|y-x|+\left|\theta_{0} x\right| \\
& \leq R+\theta_{0}(1-R)
\end{aligned}
$$

então $y \in B^{\theta_{0}}$.

Assim $B$ esta contido em alguma bola de $\vartheta^{0}$; como $B \in \vartheta$ foi escolhido arbitrariamente, então $\vartheta \subset \vartheta^{0}, \log o \vartheta=\vartheta^{0}$.

Assim $\Gamma_{0}=\cup \vartheta=\cup \vartheta^{0}$.

\subsection{1}

\section{Lemas de Crescimento}

Estes lemas levam esse nome pois permitem deduzir a partir de propriedades de positividade de $u$ (pode ser $u \geq 1$ ) sobre partes de medidas positivas de uma bola $x_{0}+B_{R / 2}$ (ou uma bola $x_{0}+\varepsilon B_{R / 2}$ ) propriedades de positividade $(u \geq \beta>0)$ sobre a toda a bola $x_{0}+B_{R / 2}$.

Utilizando o Teorema 3.1, provaremos o seguinte lema.

Lema 3.3.5 Seja $u \in W^{2, n}\left(x_{0}+B_{R}\right), 0<R \leq 1, u \geq 0$ em $x_{0}+B_{R}$ Lu $\leq 0$ q.s em $x_{0}+B_{R}$. Suponha que $\beta_{0}, 0<\beta_{0}<1$ é dado. Então para a constante $\mu_{0}=\mu_{0}\left(d, \lambda, \beta_{0}\right)=1-\left(\frac{\lambda\left(1-\beta_{0}\right)}{16(n+1) A}\right)^{n}<1$ onde $A=A(n, \lambda)$ é a constante do Teorema 3.1, da relação

$$
\left|\left(x_{0}+B_{R}\right) \cap\{u \geq 1\}\right| \geq \mu_{0}\left|B_{R}\right|,
$$

tem-se

$$
\inf _{x_{0}+B_{R / 2}}\{u\} \geq \beta_{0}
$$

Demonstração. Mediante a transformação $x \rightarrow R^{-1}\left(x-x_{0}\right)$ a bola $x_{0}+B_{R}$ se transforma em $B$.

Provaremos o Lema para $x_{0}=1, R=1$. Seja $x_{*} \in \bar{B}_{1 / 2}$ o ponto para o qual

$$
u\left(x_{*}\right)=\min _{\bar{B}_{1 / 2}}\{u\}=\inf _{B_{1 / 2}}\{u\} \text { (existe pois } u \in C^{0}\left(\bar{B}_{1}\right) \text { e } \bar{B}_{1 / 2} \text { é compacto). }
$$

Considere o caso $u\left(x_{*}\right)<1$. Seja $v(x)=1-4\left|x-x_{*}\right|^{2}-u(x)$, como $u \in C^{0}\left(\bar{B}_{1}\right) \cap W^{2, n}\left(B_{1}\right) \Rightarrow v \in C^{0}\left(\bar{B}_{1}\right) \cap W^{2, n}\left(B_{1}\right)$

Também, $V\left(x_{*}\right)=1-u\left(x_{*}\right)>0$. 
Seja $\Omega$ a componente conexa de $B_{1} \cap\{V>0\}$ contendo o ponto $x_{*}$. Também se $x \in \partial\left(x_{*}+B_{1 / 2}\right)$, então

$$
V(x)=1-4\left|x-x_{*}\right|^{2}-u\left(x_{*}\right) \leq 1-4\left|x-x_{*}\right|^{2}=0 .
$$

Tem-se

$$
\Omega \subset x_{*}+B_{1 / 2} \subset B_{1} \text { e } V=0 \text { em } \partial \Omega
$$

Também

$$
L\left(V-\left(1-4\left|x-x_{*}\right|^{2}\right)\right)=L(-u(x))=-L(u) \geq 0 .
$$

Consequentemente

$$
L(V) \geq L\left(1-4\left|x-x_{*}\right|^{2}\right)=f,
$$

em que $f=-c-4\left[2+r\left(a^{i j}\right)+2 B^{i}\left(x^{i}-x_{*}^{i}\right)-c\left|x-x_{*}\right|^{2}\right]$. Pelo Teorema 3.1 aplicado a $V$ em $\Omega, V>0$ em $\Omega, V=0$ em $\partial \Omega$, temos

$$
\max \{V\} \leq A\left\|(L V)_{-}\right\|_{L_{n}(\Omega)} .
$$

Também

$$
V\left(x_{*}\right) \leq \max \{V\}
$$

e de (3.19), temos $-L(V) \leq-f$. Devido a este último, $\max \{-L(V), 0\} \leq$ $\max \{-f, 0\}$. Então,

$$
|(L(V))|_{L_{n}(\Omega)} \leq\left|f_{-}\right|_{L_{n}(\Omega)} .
$$

Logo de (3.20)-(3.22), temos

$$
V\left(x_{*}\right) \leq A\left|f_{-}\right|_{L_{n}(\Omega)},
$$

onde $A=A(n, \lambda)$. Também de (3.18) para $x \in \Omega$, tem-se

$$
\operatorname{tr}\left(a^{i j}\right) \leq n \lambda^{-1}(\text { basta tomar } \xi=(0,0, \underbrace{1}_{i \text {-ésimo }}, \ldots, 0))
$$

Logo $a^{i j} \leq \lambda^{-1}$, de onde $\operatorname{tr}\left(a^{i j}\right)=\sum_{i=1}^{n} a^{i j} \leq d \lambda^{-1}$; de (3.18) temos que $\left|x-x_{*}\right| \leq \frac{1}{2}, \operatorname{logo}\left|B^{i}\left(x^{i}-x_{*}^{i}\right)\right| \leq|B|\left|x-x_{*}\right| \leq \frac{1}{2} \lambda^{-1}$ e $0 \leq c \leq \lambda^{-1}$. Assim

$$
\begin{aligned}
-f & =c+4\left(2 \operatorname{tr}\left(a^{i j}\right)+2 B^{i}\left(x^{i}-x_{*}^{i}\right)-c\left|x-x_{*}\right|^{2}\right) \\
& \leq \lambda^{-1}+4\left(2 n \lambda^{-1}\right)+2 \frac{1}{2} \lambda^{-1}+\frac{1}{4} \lambda^{-1} \\
& =\lambda^{-1} 8(n+1) .
\end{aligned}
$$

Assim de (3.22)

$$
V\left(x_{*}\right) \leq A\left(\int_{\Omega}\left|\lambda^{-1}(8 n+2)\right|^{n}\right)^{1 / n} .
$$

Como $V>0$, então $1-u(x)-4\left|x-x_{*}\right|^{2}>0$, logo

$$
1-u(x)>4\left|x-x_{*}\right|^{2}>0, \text { e isto implica } 1>u(x)
$$


Logo $D \subset\{u<1\}$. Como $B_{1}=B_{1} \cap\{u \geq 1\} \cup B_{1} \cap\{u<1\}$, temos

$$
\begin{aligned}
\left|B_{1}\right|= & \left|B_{1} \cap\{u \geq 1\}\right| \\
& =\left|B_{1}\right|-\left|B_{1} \cap\{u \geq 1\}\right| \\
& \leq\left|B_{1}\right|-\mu_{0}\left|B_{1}\right| \\
& =\left(1-\mu_{0}\right)\left|B_{1}\right|, \operatorname{por}(3.17) \\
& \leq 2^{n}\left(1-\mu_{0}\right) .
\end{aligned}
$$

Assim de (3.23) e (3.24)

$$
\begin{aligned}
V\left(x_{*}\right) & \leq A(8(n+1)) 2\left(1-\mu_{0}\right)^{1 / n} \\
& \leq 1-\beta_{0},
\end{aligned}
$$

então

$$
\beta_{0} \leq 1-V\left(x_{0}\right)
$$

Consequentemente,

$$
\inf _{B_{1 / 2}}\{u\}=u\left(x_{*}\right)=1-V\left(x_{*}\right) \geq \beta_{0}
$$

Fazendo uso do Principío do Máximo, provaremos o seguinte lema.

Lema 3.3.6 Seja $u \in W^{2, n}\left(x_{0}+B_{R}\right), 0<R \leq 1,0<\varepsilon \leq 1 / 2, u \geq 0 \mathrm{em}$ $x_{0}+B_{R}, L u \leq 0$ q.s em $x_{0}+B_{R}, e$

$$
\inf _{x_{0}+B_{\varepsilon R}}\{u\} \geq 1
$$

Então

$$
\inf _{x_{0}+B_{R / 2}}\{u\} \geq \varepsilon^{m}
$$

onde $m=(n+1) \lambda^{-2}>0$.

Demonstração. Fazendo a transformação $x \rightarrow R^{-1}\left(x-x_{0}\right)$ verificamos que $x_{0}+B_{R}$ transforma-se em $B_{1}$, assim o problema se reduz ao caso $x_{0}=0 \mathrm{e}$ $R=1$.

Seja $\varphi(x)=|x|^{-m}$, para $x \in \bar{B}_{1} \backslash \bar{B}_{\varepsilon}$ tem-se

$$
L \varphi=\frac{m(m+2) a^{i j} x^{i} x^{j}}{|x|^{4}} \varphi-\frac{m t r\left(a^{i j}\right)}{|x|^{2}}-\frac{m B^{i}}{|x|^{2}} \varphi-c \varphi .
$$

Para $x \in B_{1}$, por (3.18) tem-se $a^{i j} x^{i} x^{j} \geq \lambda|x|^{2}, \operatorname{tr}\left(a^{i j}\right) \leq n \lambda^{-1}$ (como no Lema 
anterior ) $\left|B^{i} x^{i}\right| \leq|B||x| \leq \lambda^{-1}, 0 \leq c \leq \lambda^{-1}$, assim

$$
\begin{aligned}
& L(\varphi-1) \geq\left(m(m+2) \lambda-m n \lambda^{-1}-m \lambda^{-1}-\lambda^{-1}\right) \frac{\varphi}{|x|^{2}} \\
& =\left[(m+2)(n+1) \lambda^{-2} \lambda-\lambda^{-1}(m n+m+1)\right] \frac{\varphi}{|x|^{2}} \\
& =\left[(2 n+1) \lambda^{-1}\right] \frac{\varphi}{|x|^{2}}
\end{aligned}
$$

Observe que na primeira desigualdade estamos utilizando o fato de que

$$
-c=-\frac{c}{|x|^{2}} \varphi \frac{|x|^{2}}{\varphi}=-\frac{c \varphi}{|x|^{2}}|x|^{2+m} \leq-\frac{c \varphi}{|x|^{2}} \leq-\lambda^{-1} \frac{\varphi}{|x|^{2}} .
$$

$\log \mathrm{O}$

$$
L u \leq 0 \leq L(\varphi-1) \text { q.s em } \bar{B}_{1} \backslash B_{\varepsilon},
$$

e também sobre $\partial B_{1} \cup \partial B_{\varepsilon}=\partial\left(B_{1} \backslash \bar{B}_{\varepsilon}\right)$.

Temos a seguinte igualdade,

$$
\varepsilon^{m}(\varphi-1)=\varepsilon^{m}\left(\frac{1}{|x|^{m}}-1\right)=\left|\frac{\varepsilon}{x}\right|^{m}-\varepsilon^{m}
$$

$\operatorname{como~inf}_{B_{\varepsilon}}\{u\} \geq 1$, então $u \geq 1$ em $\partial B_{\varepsilon}$.

Logo,

$$
u \geq \varepsilon^{m}(\varphi-1) \text { em } \partial B_{1} \cup \partial B_{\varepsilon} .
$$

Pelo Corolário 3.2 (Principio do Máximo), temos

$$
u \geq \varepsilon^{m}(\phi-1) \text { em } \bar{B}_{1} \backslash B_{\varepsilon}
$$

e também pela hipótese

$$
u \geq 1>\varepsilon^{m} \forall x \in B_{\varepsilon},
$$

e também em $\bar{B}_{1 / 2} \backslash B_{\varepsilon}, \varepsilon<|x| \leq 1 / 2$.

Logo, $\frac{1}{\varepsilon^{m}}>\frac{1}{|x|^{m}}>2^{m}, \varphi-1>2^{m}-1>1$.

Assim de (3.26), temos que

$$
u \geq \varepsilon^{m} \text { em } \bar{B}_{1 / 2} \backslash B_{\varepsilon} .
$$

De $(3.27)$ e (3.28),

$$
\inf _{B_{1 / 2}}\{u\} \geq \varepsilon^{m}
$$

Agora fazendo uso de todos os resultados vistos até agora, temos o seguinte resultado. 
Lema 3.3.7 Seja $u \in W^{2, n}\left(x_{0}+B_{R}\right), 0<R \leq 1, u \geq 0$ em $x_{0}+B_{R}, L u \leq 0$ (q.s., em $x_{0}+B_{R}$ ). Dado $\mu$ com $0<\mu<1$, existe uma constante $\beta=\beta(n, \lambda, \mu)$ tal que a relação

$$
\left|\left(x_{0}+B_{R / 2}\right) \cap\{u \geq 1\}\right| \geq \mu\left|B_{R / 2}\right|
$$

implica

$$
\inf _{x_{0}+B_{R / 2}}\{u\} \geq \beta
$$

onde $\beta$ pode ser tomado como a constante $\mathcal{H}^{k_{0}}$, onde $\mathcal{H}=2^{-(2 m+1)}$, $k_{0}=\left[\log _{\theta} \mu^{-1}\right]+1, \theta=\left[\frac{\lambda}{96(n+1) A}\right]+1, A=A(n, \lambda)$ são as constantes do Teorema 3.1.

Corolário 3.3 Seja $u \in W^{2, n}\left(B_{R}\right), 0<R \leq 1, L u \geq 0$ q.s, em $B_{R}$. Dado $\mu$ com $0<\mu<1$, da relação

$$
\left|B_{R / 2} \cap\{u \leq 0\}\right| \geq \mu\left|B_{R / 2}\right|
$$

tem-se,

$$
\sup _{B_{R}}\{u\} \geq M \sup _{B_{R / 2}}\{u\},
$$

onde $M=M(n, \lambda, \mu)=(1-\beta)^{-1}>1$, e $\beta$ é a constante do Lema 3.3.7.

Demonstração. Sem perda de generalidade, suponha que $\sup _{B_{R}}\{u\}>0$. Então com a transformação $u \rightarrow N u$, com $N>0$ conveniente a demonstração se reduz a

$$
\sup _{B_{R}}\{u\}=1
$$

Seja $v=1-u$, com $u \leq 1$ então $v \geq 0$ em $B_{R}, L v=-c-L u \leq 0$, e $\left|B_{R / 2} \cap\{v \geq 1\}\right|=\left|B_{R / 2} \cap\{u \leq 0\}\right| \geq \mu\left|B_{R / 2}\right|$.

Logo pelo Lema 3.3.7

$$
\inf _{B_{R / 2}}\{v\} \geq \beta, \text { onde } \beta=\beta(n, \lambda, \mu)>0 .
$$

Logo como $\sup _{B_{R / 2}}\{u\}=\sup _{B_{R / 2}}\{1-v\}=1-\inf _{B_{R}}\{v\}$, então

$$
\sup _{B_{R / 2}}\{u\} \leq 1-\beta=(1-\beta) \sup _{B_{R / 2}}\{u\},
$$

de onde $\sup _{B_{R}}\{u\} \geq \underbrace{(1-\beta)^{-1}}_{M} \sup _{B_{R / 2}}\{u\}$.

O seguinte resultado será de muita utilidade na demonstração da Desigualdade de Harnack. Na sua prova utilizaremos o lema 3.4 e o lema 3.6. 
Lema 3.3.8 Seja $u \in W^{2, n}\left(x_{0}+B_{R}\right), 0<R \leq 1, u \geq 0$ em $x_{0}+B_{R}, L u=0$ q.s em $B_{R}$. Suponha $u\left(x_{0}\right) \geq 1$ e que para alguma constante $M>1$ tem-se $\sup _{x_{0}+B_{R / 2}}\{u\} \leq M$. Então

$$
\inf _{x_{0}+B_{R}}\{u\} \geq \beta_{1}>0,
$$

onde $\beta_{1}=\beta_{1}(n, \lambda, M)=\frac{1}{2} \beta>0$, sendo $\beta$ a constante do Lema 3.3 .7 calculada para $\mu=\left[\frac{\lambda}{16(n+1)(2 M-1) A}\right]^{n}$.

Demonstração. Suponha $x_{0}=0$ e seja $v=\frac{M-u}{M-1 / 2}$, tem-se $v \geq 0$ em $B_{R / 2}$ (pois $u \leq M$ em $B_{R}$ ) e $L v=\frac{L(M)-L u}{M-1 / 2}=\frac{-c M-0}{M-1 / 2} \leq 0$ q.s em $B_{R / 2}$ (pois $c \geq 0$ ), então

$$
\inf _{B_{R / 4}}\{v\} \leq v(0)=\frac{M-u(0)}{M-1 / 2}<\frac{M-1}{M-1 / 2}<1 .
$$

Aplicando o Lema 3.3.5 (na sua forma contra reciproca) a $v$ e à bola $B_{R / 2}$, com $\mu_{0}=\mu_{0}\left(n, \lambda, \frac{M-1}{M-1 / 2}\right)=1-\left[\frac{\lambda}{16(n+1)(2 M-1) A}\right]^{n}$, tem-se

$$
\begin{aligned}
& \left|B_{R / 2} \cap\{v \geq 1\}\right|<\mu_{0}\left|B_{R / 2}\right|, \\
& \text { i.e., } \\
& \left|B_{R / 2} \cap\{v \leq 1\}\right| \geq\left(1-\mu_{0}\right)\left|B_{R / 2}\right|,
\end{aligned}
$$

então

$$
\left|B_{R / 2} \cap\{u \geq 1 / 2\}\right|=\left|B_{R / 2} \cap\{v \leq 1\}\right| \geq\left(1-\mu_{0}\right)\left|B_{R / 2}\right| .
$$

Pelo Lema 3.3.7 com $\mu=1-\mu_{0}$, tem-se

$$
\inf _{B_{R / 2}}\{u\} \geq \frac{1}{2} \beta\left(n, \lambda, 1-\mu_{0}\right)=\beta_{1} .
$$

\subsection{2}

\section{Desigualdade de Harnack para Equações Elípticas}

A Desigualdade de Harnack diz que para soluções em $W^{2, n}\left(B_{R}\right)$ de $L u=0$ em $B_{R}$ com $u \geq 0$ em $B_{R}$ tem-se que $\sup \left\{u, B_{R / 8}\right\} \leq N \operatorname{Ninf}\left\{u, B_{R / 8}\right\}$, onde $N$ depende da dimensão do espaço e do operador $L$. Mais exatamente, temos os seguinte resultado.

Teorema 3.4 Suponha que os coeficientes de L em (3.1) satisfazem as condições (3.2) e que $u \in W^{2, n}\left(B_{R}\right), 0<R \leq 1, u \geq 0$ em $B_{R}$, Lu=0 q.s em $B_{R}$. Então,

$$
\sup _{B_{R / 8}}\{u\} \leq N \inf _{B_{R / 8}}\{u\}
$$


onde $N=N(n, \lambda)=12^{m} \beta_{1}^{-1}, m=(n+1) \lambda^{-2}, \beta_{1}$ é a constante do Lema 3.3.8, tomado quando $M=2^{m}$. No caso particular $B=0, c=0 \Rightarrow$, a desigualdade (3.30) vale para $R \geq 1$.

Demonstração. Fazendo a transformação $x \rightarrow R^{-1} x, u \rightarrow C u$ com uma constante $C$ apropriada, reduzimos o problema ao caso $R=1 \mathrm{e}$

$$
\sup _{B_{1 / 8}}\{u\}=1
$$

Para $\frac{1}{8} \leq \tau<\frac{1}{4}$ e $\left(m=(n+1) \lambda^{-2}\right.$, definimos as funções

$$
\eta(\tau)=\max _{\bar{B}_{\tau}}\{u\}, \nu(\tau)=(2-8 \tau)^{-m} .
$$

Seja $\tau_{0}$ a maior raiz de $\eta(\tau)=\nu(\tau)$. Como

$$
\eta\left(\frac{1}{8}\right)=1=\nu\left(\frac{1}{8}\right), \lim _{\tau \rightarrow 1 / 4} \nu(\tau)=+\infty
$$

e $u$ é continua e limitada em $B_{1 / 4}$, segue que $\tau_{0}$ é corretamente definido e $\tau_{0}<1 / 4$.

Para algum $x_{1} \in \bar{B}_{\tau_{0}}$, tem-se $u\left(x_{1}\right)=\eta\left(\tau_{0}\right)=\nu\left(\tau_{0}\right)$. Se $\tau_{1}=\frac{1-4 \tau_{0}}{8}$, como $x_{1} \in B_{\tau_{0}}$, temos

$$
x_{1}+B_{\tau_{1}} \subset \frac{B_{1}+4 \tau_{0}}{8} \text {, pois } \tau_{0}+\underbrace{\frac{1-4 \tau_{0}}{8}}_{\tau_{1}}=\frac{1+4 \tau_{0}}{8} .
$$

Por tanto da definição de $\tau_{0}$, temos

$$
\sup _{x_{1}+B_{\tau_{1}}}\{u\} \leq \eta\left(\frac{1+4 \tau_{0}}{8}\right)<\nu\left(\frac{1+4 \tau_{0}}{8}\right)=\left(1-4 \tau_{0}\right)^{-m}=2^{m} \nu\left(\tau_{0}\right) .
$$

Aplicando o Lema 3.3.8 a função $\frac{u}{\nu\left(\tau_{0}\right)}$ (note que $\left(x_{0}+B_{\tau_{0}}=x_{1}+B_{2 \tau_{1}} \subset\right.$ $\left.B_{1 / 4}, M=2^{m}\right)$ ) tem-se

$$
\inf _{x_{1}+B_{\tau_{1}}}\left\{\frac{u}{\nu\left(\tau_{0}\right)}\right\} \geq \beta_{1} \Rightarrow \inf _{x_{1}+B_{\tau_{1}}}\{u\} \geq \beta_{1} \nu\left(\tau_{0}\right),
$$

em que $\beta_{1}=\beta_{1}\left(n, \lambda, 2^{m}\right)>0$.

Finalmente observe que

$$
x_{1} \in \bar{B}_{\tau_{0}} \subset B_{1 / 4}, B_{1 / 8} \subset x_{1}+B_{3 / 4} \subset B_{1} .
$$


Pelo Lema 3.3.6, $x_{0}+B_{\tau}=x_{1}+B_{3 / 4}, \mu=\frac{4}{3} \tau, \log \mathrm{O}$,

$$
\begin{aligned}
\inf _{B_{1 / 8}}\{u\} & \geq \inf _{x_{1}+B_{3 / 8}}\{u\} \\
& \geq \beta_{1} \nu\left(\tau_{0}\right)\left(\frac{3}{4} \tau_{1}\right)^{m} \\
& =\beta_{1}\left(2-8 \tau_{0}\right)^{-m}\left(\frac{1-4 \tau_{0}}{6}\right)^{m} \\
& =12^{-m}=N^{-1} \cdot 1=N^{-1} \sup _{B_{1 / 8}}\{u\}
\end{aligned}
$$

ficando provado o Teorema.

Corolário 3.5 (Teorema unilateral de Liuoville)

Suponha que $B=0$ e $c=0, u \in W^{2, n}\left(B_{R}\right)$ para todo $R>0, u \geq 0$ em $\mathbb{R}^{n}$ e $L u=0$ q.s em $\mathbb{R}^{n}$. Então u é constante.

Demonstração. Considerando $\bar{u}=u-\inf _{\mathbb{R}^{n}}\{u\}$ tem-se que $\inf _{\Omega} \bar{u}=0$, logo a desigualdade (3-29) vale para todo $R>0$. Logo,

$$
\sup _{B_{R / 8}}\{\bar{u}\} \leq N \inf _{B_{R / 8}} \bar{u}=0
$$

assim se $R \rightarrow \infty, \bar{u}=0$ em $\mathbb{R}^{n}$ e $\operatorname{logo} \bar{u}=u-\inf _{\mathbb{R}^{n}}\{u\}=0$, então

$$
u=\inf _{\mathbb{R}^{n}}\{u\}=c t e
$$

\subsection{3}

\section{Propriedades de Hölder da Solução da Equação Elíptica}

A Propriedade de Holder consiste em provar estimativas sobre uma norma de Hölder de $u$ sobre um sub-domínio $\Omega^{\prime}$ em termos de $\sup \{|u|, \Omega\}$ e $\|L u\|_{L_{\Omega}(\Omega)}$. Como antes, consideremos os operadores elípticos (3.1) com coeficientes que satisfazem as condições (3.2).

Lema 3.3.9 Suponha que $u \in W^{2, n}\left(B_{R}\right), 0<R \leq 1, C=0, L u=0$ q.s em $B_{R}$ então para todo $x_{0}, x_{1} \in B_{R}$ tem-se

$$
\left|u\left(x_{0}\right)-u\left(x_{1}\right)\right| \leq N R^{-\alpha}\left|x_{0}-x_{1}\right|^{\alpha} \sup _{B_{R}}\{|u|\},
$$

onde $N=N(n, \lambda)=2 \nu, \alpha=\alpha(n, \lambda)=\log _{2} \nu, \nu=\frac{1+M}{2}>1$, e $M=M(n, \lambda)>1$ é a constante do Corolário 3.2. 
Demonstração. Fazendo a transformação $x \rightarrow R^{-1} x$ reduzimos a demonstração ao caso $R=1$. Usando a bibliografia [5], pag 59-60, bastará estabelecer a estimativa

$$
o s c_{x+B_{R}}\{u\} \geq \nu \cdot o s c_{x+B_{\tau / 2}}\{u\}
$$

para cada bola $x+B_{\tau / 2} \subset B_{1}$, onde $\operatorname{osc}_{\Gamma}\{u\}=\sup _{\Gamma}\{u\}-\inf _{\Gamma}\{u\}$.

Também usando argumentos de $[3,5]$ que consideram $c_{1} u+c_{2}$ em lugar de $u$. Para constante adequada $c_{1}$ e $c_{2}$ reduzimos a prova de (3.31) ao caso

$$
\begin{aligned}
& \sup _{x+B_{\tau / 2}}\{u\}=1, \inf _{x+B_{\tau / 2}}\{u\}=-1, \\
& \left|\left(x+B_{\tau / 2}\right) \cap\{u \leq 0\}\right| \geq \frac{1}{2}\left|B_{\tau / 2}\right| .
\end{aligned}
$$

Aplicando neste caso o Corolário 3.3 com $\mu=1 / 2$ tem-se,

$$
\sup _{x+B_{\tau}}\{u\} \geq M \sup _{x+B_{\tau / 2}}\{u\}=M
$$

onde $M=M(n, \lambda, 1 / 2)>1$ é a constante do Corolário 3.3.

Agora,

$$
\inf _{x+B_{\tau}}\{u\} \leq \inf _{x+B_{\tau / 2}}\{u\}=-1 \Rightarrow-\inf _{x+B_{\tau}}\{u\} \geq 1 .
$$

Assim de (3.32) e (3.33)

$$
o s c_{x+B_{\tau}}\{u\}=\sup _{x+B_{\tau / 2}}\{u\}-\inf _{x+B_{\tau}}\{u\} \geq M+1
$$

enquanto

$$
\operatorname{osc}_{x+B_{\tau / 2}}\{u\}=\underbrace{\sup _{x+B_{\tau / 2}}\{u\}}_{1}-\underbrace{\inf _{x+B_{\tau / 2}}\{u\}}_{-1}=2 .
$$

Logo tem-se (3.31) com $\nu=\frac{1+M}{2}>1$ pois $\operatorname{osc}_{x+B_{\tau}}\{u\} \geq M+1=\frac{M+1}{2}-2=$ $\frac{M+1}{2} \operatorname{osc}_{x+B_{\tau / 2}}\{u\}$, e o Lema fica provado.

Lema 3.3.10 Seja $u \in W^{2, n}\left(B_{R}\right), 0<R \leq 1, c=0$. Então para $x_{0}, x_{1} \in$ $\bar{B}_{R / 2}$, temos

$$
\left|u\left(x_{0}\right)-u\left(x_{1}\right)\right| \leq N_{1}\left[R^{-\alpha}\left|x_{0}-x_{1}\right|^{\alpha} \sup _{B_{R}}\{|u|\}+R|L u|_{L^{n}\left(B_{R}\right)}\right],
$$

onde $N_{1}=N_{1}(n, \lambda)=\max \{N, A\}, N$ e $\alpha$ são as constantes do Lema 3.3 .9 e A é a constante do Teorema 3.1.

Demonstração. Em (3.34) aplicamos um processo de limite com funções regulares $u$ e operadores $L$ com coeficientes regulares. Assim para $u, L$ arbitrários podemos supor que $u$ e os coeficientes de $L$ são regulares. Então $u=u_{1}+u_{2}$ 
são funções regulares, soluções dos problemas

$$
\left\{\begin{array}{l}
L u_{1}=0 \text { em } B_{R} \\
u_{1}=u \text { em } \partial B_{R}
\end{array}\right.
$$

e

$$
\left\{\begin{array}{l}
L u_{2}=L u \text { em } B_{R} \\
u_{2}=0 \text { em } \partial B_{R}
\end{array}\right.
$$

Pelo Principio do Máximo tem-se

$$
\sup _{B_{R}}\left|u_{1}\right| \leq \sup _{\partial B_{R}}\left|u_{1}\right|=\sup _{\partial B_{R}}|u| \leq \sup _{B_{R}}|u|
$$

Também pelo Lema 3.3.9, aplicado a $u$, tem-se

$$
\begin{aligned}
\left|u_{1}\left(x_{0}\right)-u_{1}\left(x_{1}\right)\right| & \leq N R^{-\alpha}\left|x_{0}-x_{1}\right|^{\alpha} \sup _{B_{R}}\left\{\left|u_{1}\right|\right\} \\
& \leq N R^{-\alpha}\left|x_{0}-x_{1}\right|^{-\alpha} \sup _{B_{R}}\{|u|\},
\end{aligned}
$$

e pelo Teorema 3.1 aplicado a $u_{2}$ tem-se,

$$
\begin{aligned}
\max _{B_{R}}\left\{u_{2}\right\} & \leq A R\left\|L u_{2-}\right\|_{L^{n}\left(B_{R}\right)} \\
& \leq A R\left|\left(L u_{-}\right)\right|_{L^{n}\left(B_{R}\right)} \\
& \leq A R|L u|_{L^{n}\left(B_{R}\right)} .
\end{aligned}
$$

Logo,

$$
\begin{gathered}
\left|u_{2}\left(x_{0}\right)-u_{2}\left(x_{1}\right)\right| \leq \max \left\{u_{2}\right\}\left(\text { pois } u_{2}>0\right) \\
\leq A R|L u|_{L^{n}\left(B_{R}\right)} .
\end{gathered}
$$

De (3.35) e (3.36), tem-se

$$
\begin{aligned}
\left|u\left(x_{0}\right)-u\left(x_{1}\right)\right| & =\left|\left(u_{1}+u_{2}\right)\left(x_{0}\right)-\left(u_{1}+u_{2}\right)\left(x_{1}\right)\right| \\
& \leq\left|u_{1}\left(x_{0}\right)-u_{1}\left(x_{1}\right)\right|+\left|u_{2}\left(x_{0}\right)-u\left(x_{1}\right)\right| \\
& \leq N R^{-\alpha}\left|x_{0}-x_{1}\right|^{-\alpha} \sup _{B_{R}}\{u\}+A R|L u|_{L^{n}\left(B_{R}\right)} \\
& \leq \underbrace{(N \vee A)}_{N_{1}}\left[\left|x_{0}-x_{1}\right|^{-\alpha} \sup _{B_{R}}\{u\}+R|L u|_{L^{n}\left(B_{R}\right)}\right],
\end{aligned}
$$

ficando o Lema provado.

Para os próximos Teoremas, $\alpha=\alpha(\xi, \lambda)>0$ é a constante do Lema 3.3.9, $\aleph=\frac{\alpha}{1+\alpha}>0, \Omega$ é um domínio de $\mathbb{R}^{n}$ e para $x \in \bar{\Omega}$ denota-se $\rho(x)=\rho(x, \partial \Omega)=\inf \{|x-y|: y \in \partial \Omega\}$.

Teorema 3.6 Seja $u \in W^{2, n}(\Omega), x_{0}, x_{1} \in \Omega, 2\left|x_{0}-x_{1}\right| \leq \rho\left(x_{0}\right), B=0, C=$ 0. Então para todo $\rho \in\left[2\left|x_{0}-x_{1}\right|, \rho\left(x_{0}\right)\right]$, tem-se

$$
\left|u\left(x_{0}\right)-u\left(x_{1}\right)\right| \leq 2 N_{1} \rho^{-\aleph}\left|x_{0}-x_{1}\right|^{\aleph}\left[\sup _{\Omega}\{|u|\}+\rho|L u|_{L^{n}(\Omega)}\right],
$$


onde $N_{1}=N_{1}(n, \lambda)$ é a constante do Lema 3.3.10.

Demonstração. Seja $\tau=2^{\aleph} \rho^{1-\aleph}\left|x_{0}-x_{1}\right|^{\aleph}, \aleph=\frac{\alpha}{1+\alpha}$ e do fato de que

$$
2\left|x_{0}-x_{1}\right| \leq \rho \leq \rho\left(x_{0}\right)
$$

temos

$$
\begin{aligned}
\tau & =2^{\frac{\alpha}{1+\alpha}} \underbrace{(\inf \{|x-y| / y \in \partial \Omega\})^{\frac{1}{1+\alpha}}}_{\rho}\left|x_{0}-x_{1}\right|^{\frac{\alpha}{1+\alpha}} \\
& \leq 2^{\frac{\alpha}{1+\alpha}} \rho^{\frac{1}{1+\alpha}}\left(\frac{\rho}{2}\right)^{\frac{\alpha}{1+\alpha}} \\
& =\rho .
\end{aligned}
$$

Também

$$
\begin{aligned}
\tau & \geq 2^{\frac{\alpha}{1+\alpha}}\left(2\left|x_{0}-x_{1}\right|\right)^{\frac{1}{1+\alpha}}\left|x_{0}-x_{1}\right|^{\frac{\alpha}{1+\alpha}} \\
& =2\left|x_{0}-x_{1}\right| .
\end{aligned}
$$

Assim tem-se

$$
2\left|x_{0}-x_{1}\right| \leq \tau \leq \rho \leq \rho\left(x_{0}\right)
$$

Logo para todo $z \in x_{0}+B_{\tau},\left|z-x_{0}\right| \leq \tau \leq \rho\left(x_{0}\right) \Rightarrow x_{0}+B_{\tau} \subset \Omega \mathrm{e}$ $\left|x_{0}-x_{1}\right| \leq \frac{\tau}{2} \Rightarrow x_{1} \in x_{0}+\bar{B}_{\tau / 2}$.

Assim, pelo Lema 3.3.10 aplicado a $x_{0}+\bar{B}_{\tau / 2}$ tem-se

$$
\begin{aligned}
\left|u\left(x_{0}\right)-u\left(x_{1}\right)\right| & \leq N_{1}\left[\tau^{-\alpha}\left|x_{0}-x_{1}\right|^{\alpha} \sup _{x_{0}+B_{\tau / 2}}\{|u|\}+\tau|L u|_{L^{n}\left(x_{0}+B_{\tau}\right)}\right] \\
& =N_{1}\left[2^{\aleph(-\alpha)} \rho^{(1-\aleph)(-\alpha)}\left|x_{0}-x_{1}\right|^{\aleph(-\alpha)}\left|x_{0}-x_{1}\right|^{\alpha} \sup _{x_{0}+B_{\tau / 2}}\{|u|\}+\tau|L u|_{L^{n}\left(x_{0}+B_{\tau}\right)}\right]
\end{aligned}
$$

e como

$$
\left\{\begin{array}{l}
(1-\aleph)(-\alpha)=\frac{1}{1+\alpha}(-\alpha)=-\aleph \\
\aleph(-\alpha)+\alpha=\alpha(1-\aleph)=\alpha\left(\frac{1}{1+\alpha}\right)=\aleph
\end{array}\right.
$$

tem-se,

$$
\begin{aligned}
\left|u\left(x_{0}\right)-u\left(x_{1}\right)\right| & \leq N_{1}\left[2^{-\alpha \aleph} \rho^{-\aleph}\left|x_{0}-x_{1}\right|^{\aleph} \sup _{x_{0}+B_{\tau / 2}}\{|u|\}+2^{\aleph} \rho^{1-\aleph}\left|x_{0}-x_{1}\right||L u|_{L^{n}\left(x_{0}+B_{\tau}\right)}\right] \\
& \leq 2 N_{1} \rho^{-\aleph}\left|x_{0}-x_{1}\right|^{\aleph}\left[\sup _{x_{0}+B_{\tau / 2}}\{|u|\}+2^{\aleph} \rho|L u|_{L^{n}\left(x_{0}+B_{\tau}\right)}\right] .
\end{aligned}
$$

Logo

$$
\left|u\left(x_{0}\right)-u\left(x_{1}\right)\right| \leq 2 N_{1} \rho^{-\aleph}\left|x_{0}-x_{1}\right|^{\aleph}\left[\sup _{\Omega}\{|u|\}+2^{\aleph} \rho|L u|_{L^{n}(\Omega)}\right],
$$

ficando provado o Teorema. 
Teorema 3.7 Seja $u \in W^{2, n}(\Omega), x_{0}, x_{1} \in \Omega, 2\left|x_{0}-x_{1}\right| \leq \min \left\{1, \rho\left(x_{0}\right)\right\}$, então

$$
\left|u\left(x_{0}\right)-u\left(x_{1}\right)\right| \leq N_{2}\left(1 \wedge \rho\left(x_{0}\right)\right)^{-\aleph}\left|x_{0}-x_{1}\right|^{\aleph}\left[\sup _{\Omega}\{|u|\}+|L u|_{L^{n}(\Omega)}\right]
$$

onde $N_{2}-N_{2}(n, \lambda)=2\left(1+2 \lambda^{-1}\right) N_{1}$, sendo $N_{1}$ a constante do Lema 3.3.10.

Demonstração. Seja $\rho=1 \wedge \rho\left(x_{0}\right)$, seguindo como na demonstração do Teorema anterior (que usa o Lema 3.3.10, o qual por sua vez usa o Teorema 3.1 que é valido para $L u+c u$ e o Lema 3.3.9 que pode ser adaptado a $L u+c u$ ).

Assim tem-se por (3.37) do Teorema 3.6 com lado direito $L u+c u$ em lugar de $L u$,

$$
\left|u\left(x_{0}\right)-u\left(x_{1}\right)\right| \leq 2 N_{1} \rho^{-\aleph}\left|x_{0}-x_{1}\right|^{\aleph}\left[\sup _{x_{0}+B_{\tau}}\{|u|\}+\rho|L(u+c u)|_{L^{n}\left(x_{0}+B_{\tau}\right)}\right],
$$

também,

$$
\begin{aligned}
|L u+c u|_{L^{n}\left(x_{0}+B_{\tau}\right)} & \leq|L u|_{L^{n}\left(x_{0}+B_{\tau}\right)}+|c u|_{L^{n}\left(x_{0}+B_{\tau}\right)}, \\
& \leq|L u|_{L^{n}\left(x_{0}+B_{\tau}\right)}+\lambda^{-1}\left|B_{\tau}\right|^{1 / n} \sup _{x_{0}+B_{\tau}}|u| .
\end{aligned}
$$

Daqui, temos

$$
|L u+c u|_{L^{n}\left(x_{0}+B_{\tau}\right)} \leq|L u|_{L^{n}\left(x_{0}+B_{\tau}\right)}+\lambda^{-1}\left|B_{1}\right|^{1 / n} \sup _{x_{0}+B_{\tau}}|u| .
$$

Assim de (3.38) e (3.39) tem-se

$$
\begin{aligned}
\left|u\left(x_{0}\right)-u\left(x_{1}\right)\right| & \leq 2 N_{1} \rho^{-\aleph}\left|x_{0}-x_{1}\right|^{\aleph}\left[\sup _{\Omega}\{|u|\}\left(1+\lambda^{-1}\left|B_{1}\right|^{1 / n}\right)+|L u|_{L^{n}(\Omega)}\right] \\
& \leq \underbrace{2 N_{1}\left(1+\lambda^{-1}\left|B_{1}\right|^{1 / n}\right)}_{N_{2}}\left(1 \wedge \rho\left(x_{0}\right)\right)^{-\aleph}\left|x_{0}-x_{1}\right|^{\aleph}\left[\sup _{\Omega}\{|u|\}+|L u|_{L^{n}(\Omega)}\right],
\end{aligned}
$$

ficando provado o Teorema.

Teorema 3.8 Suponha que o dominio $\Omega^{\prime} \subset \Omega, u \in W^{2, n}(\Omega)$. Denotamos $\rho=\rho\left(\Omega^{\prime}, \partial \Omega\right)=\inf \left\{|x-y|, x \in \Omega^{\prime}, y \in \partial \Omega\right\}$ e suponhamos que $\rho>0$. Então para todo $x_{0}, x_{1} \in \Omega^{\prime}$ tem-se

$$
\left|u\left(x_{0}\right)-u\left(x_{1}\right)\right| \leq N_{2}(1 \wedge \rho)^{-\aleph}\left|x_{0}-x_{1}\right|^{\aleph}\left[\sup _{\Omega}|u|+|L u|_{L^{n}(\Omega)}\right],
$$

onde $N_{2}=N_{2}(n, \lambda)$ é a constante do Teorema 3.7. 
Demonstração. Se $2\left|x_{0}-x_{1}\right| \leq(1 \wedge \rho)$, como $x_{0} \in \Omega^{\prime}$,

$$
\begin{aligned}
\rho\left(x_{0}\right) & =\rho\left(x_{0}, \partial \Omega\right) \\
& =\inf \left\{\left|x_{0}-y\right| / y \in \partial \Omega\right\} \\
& \geq \inf \left\{|x-y| / x \in \Omega^{\prime}, y \in \partial \Omega\right\} \\
& =\rho .
\end{aligned}
$$

Logo,

$$
1 \wedge \rho\left(x_{0}\right) \geq 1 \wedge \rho \Rightarrow\left(1 \wedge \rho\left(x_{0}\right)\right)^{-\aleph} \leq(1 \wedge \rho)^{-\aleph}
$$

Assim do Teorema 3.7

$$
\begin{aligned}
\left|u\left(x_{0}\right)-u\left(x_{1}\right)\right| & \leq N_{2}\left(1 \wedge \rho\left(x_{0}\right)\right)^{-\aleph}\left|x_{0}-x_{1}\right|^{\aleph}\left[\sup _{\Omega}\{|u|\}+|L u|_{L^{n}(\Omega)}\right] \\
& \leq N_{2}(1 \wedge \rho)^{-\aleph}\left|x_{0}-x_{1}\right|^{\aleph}\left[\sup _{\Omega}\{|u|\}+|L u|_{L^{n}(\Omega)}\right] .
\end{aligned}
$$

Também, se $2\left|x_{0}-x_{1}\right|>(1 \wedge \rho) \Rightarrow 2^{\aleph}\left|x_{0}-x_{1}\right|^{\aleph}(1 \wedge \rho)^{-\aleph}>1, \log 0$

$$
2^{\aleph+1}=2^{\frac{\alpha}{1+\alpha}+1}<4<2\left(1+\lambda^{-1}\right) \underbrace{(N \vee A)}_{N_{1}}=2\left(1+\lambda^{-1}\right)((1+M) \vee A)=N_{2} .
$$

Assim $2^{\aleph+1}<4<N_{2}$. Logo,

$$
\begin{aligned}
\mid u\left(x_{0}\right) & -u\left(x_{1}\right) \mid \\
& \leq 2 \sup _{\Omega}\{|u|\} \leq 2\left(2^{\aleph}\left|x_{0}-x_{1}\right|^{\aleph}(1 \wedge \rho)^{-\aleph}\right) \sup _{\Omega}\{|u|\} \\
& \leq 2^{\aleph+1}(1 \wedge \rho)^{-\aleph}\left|x_{0}-x_{1}\right|^{-\aleph} \sup _{\Omega}|u| \leq N_{2}(1 \wedge \rho)^{-\aleph}\left|x_{0}-x_{1}\right|^{\aleph} \sup _{\Omega}|u| .
\end{aligned}
$$

De (3.40) e (3.41), tem-se

$$
\left|u\left(x_{0}\right)-u\left(x_{1}\right)\right| \leq N_{2}(1 \wedge \rho)^{-\aleph}\left|x_{0}-x_{1}\right|^{\aleph}\left[\sup _{\Omega}|u|+|L u|_{L^{n}(\Omega)}\right] .
$$




\section{4}

\section{Regularidades de Equações Elípticas na Forma Divergente}

Neste capítulo consideremos o operador $L$ dado na forma divergente definido por:

$$
L u=D_{i}\left(a^{i j}(x) D_{j} u+b^{i} u\right)+c^{i}(x) D_{i} u+d(x) u,
$$

onde os coeficientes $a^{i j}, b^{i}, c^{i}, d$ são mensuráveis no domínio $\Omega \subset \mathbb{R}^{n}$. O operador $L$ é estritamente elíptico em $\Omega$, i.e,

$$
\exists \lambda \in \mathbb{R}^{+} \text {tal que } a^{i j}(x) \xi_{i} \xi_{j} \geq \lambda|\xi|^{2} \forall x \in \Omega, \xi \in \mathbb{R}^{n},
$$

e seus coeficientes são limitados: para alguns $\Lambda, \gamma \geq 0$ temos

$$
\sum_{i, j=1}^{n} a^{i j}(x) \leq \Lambda^{2} \text { e } \lambda^{-2} \sum_{i, j=1}\left(\left|D_{j} b^{i}(x)\right|^{2}+\left|D_{j} c^{i}(x)\right|^{2}\right)+\lambda^{-1}|d(x)| \leq \gamma^{2} .
$$

Nesse capítulo trabalharemos com o espaço de Sobolev $W^{1,2}(\Omega)$ definido por,

$$
W^{1,2}(\Omega)=\left\{f: \Omega \subset \mathbb{R}^{n} \mapsto \mathbb{R} / D^{\alpha} f \in L^{2}(\Omega), \alpha \in \mathbb{N}^{n}, \alpha_{1}+\cdots+\alpha_{n} \leq 1\right\}
$$

Por definição $u \in W^{2,1}(\Omega)$ é solução generalizada de

$$
\left\{\begin{array}{l}
L u=g+D_{i} f^{i} \\
u=\varphi \text { em } \partial \Omega
\end{array}\right.
$$

se satisfaz

$$
\begin{aligned}
& L(u, v)=\int_{\Omega}\left(a^{i j} D_{j} u+b^{i} u\right) D_{i} v-\left(c^{i} D_{i} u+d u\right) v d x \\
& =F(v)=\int_{\Omega} f^{i} D_{i} v-g v d x, \forall v \in C_{0}^{1}(\Omega) \\
& \varphi \in W^{1,2}(\Omega) \text { e } u-\varphi \in W_{0}^{1,2}(\Omega) .
\end{aligned}
$$

Observe que de (4.2) tem-se

$$
|L(u, v)| \leq C|| u\left\|_{W^{1,2}(\Omega)} \mid\right\| v \|_{W^{1,2}(\Omega)} .
$$

Para alguns resultados precisaremos também da seguinte hipótese 


$$
\int_{\Omega}\left(d v-b^{i} D_{i} v\right) d x \leq 0, \quad \forall v \geq 0, v \in C_{0}^{1}(\Omega)
$$

\subsection{1}

\section{Limitações Globais de Soluções Fracas}

Nesta seção derivaremos resultados de limitações globais de soluções de (4-3) em $W^{1,2}(\Omega)$ que são limitadas sobre a fronteira de $\Omega$. Usaremos as técnicas das funções testes, que não dependem tanto da linearidade do operador $L$ mas sim da estrutura não linear satisfeita por ele. Sendo mais explícito podemos escrever a equação

$$
L u=g+D_{i} f^{i}
$$

na forma

$$
\begin{gathered}
D_{i}\left(A^{i}(x, u, D u)\right)+B(x, u, D u)=0 \\
\left\{\begin{array}{c}
\text { onde } A^{i}(x, z, P)=a^{i j}(x) P_{j}+b^{i}(x) z-f^{i}(x) \\
B(x, z, P)=c^{i}(x) P_{i}+d(x) z-g(x) \\
\text { para todo }(x, z, P) \in \Omega \times \mathbb{R} \times \mathbb{R}^{n} .
\end{array}\right.
\end{gathered}
$$

Uma função fracamente diferenciável é chamada subsolução (supersolução, solução) fraca de (4.5) em $\Omega$, se $A^{i}(x, u, D u), B(x, u, D u)$ são localmente integráveis e

$$
\int_{\Omega}\left(D_{i} v A^{i}(x, u, D u)-v B(x, u, D u)\right) d x \leq 0(\geq,=0) \text { para todo } v \geq 0 \text { em } C_{0}^{1}(\Omega)
$$

Escrevendo $b=\left(b^{1}, \ldots, b^{n}\right), c=\left(c^{1}, \ldots, c^{n}\right), f=\left(f^{1}, \ldots, f^{n}\right)$ e usando (4.1) e desigualdade de Schwartz tem-se

$$
\left\{\begin{array}{l}
P_{i} A^{i}(x, z, P) \geq \frac{1}{2}|P|^{2}-\frac{1}{\lambda}\left(|b z|^{2}+|f|^{2}\right) \\
|B(x, z, P)| \leq|c||P|+|d z|+|g|
\end{array}\right.
$$

com efeito, de (4.1) tem-se

$$
(i)\left\{\begin{aligned}
P_{i} A^{i}(x, z, P) & =P_{i}\left(a^{i j}(x) P_{j}+b^{i} z-f^{i}(x)\right) \\
& \geq \lambda|P|^{2}+P_{i} b^{i} z-P_{i} f^{i}
\end{aligned}\right.
$$

também

$$
\begin{aligned}
& \left|P_{i} b^{i} z\right| \leq \frac{\lambda}{4}|P|^{2}+\frac{1}{\lambda}|b z|^{2} \\
& \left|P_{i} f^{i}\right| \leq \frac{\lambda}{4}|P|^{2}+\frac{1}{\lambda}|f|^{2}
\end{aligned}
$$

Logo

$$
\text { (ii) } P_{i} b^{i} z-P_{i} f^{i} \geq \frac{\lambda}{2}|P|^{2}-\frac{1}{\lambda}\left(|b z|^{2}+|f|^{2}\right),
$$

assim de $(i)$ e $(i i)$ tem-se $(4.7)_{1}$, também 


$$
\begin{aligned}
|B(x, z, P)| & =\left|c^{i} P_{i}+d z-g\right| \\
& \leq|c||P|+|d z|+|g|,
\end{aligned}
$$

de onde tem-se $(4.7)_{2}$.

Escrevendo, para algum $k>0$,

$$
\bar{z}=|z|+k, \bar{b}=\frac{1}{\lambda^{2}}\left(|b|^{2}+|c|^{2}+\frac{1}{k^{2}}|f|^{2}\right)+\frac{1}{\lambda}\left(|d|+\frac{1}{k}|g|\right)
$$

tem-se

$$
\text { (iv) } P_{i} A^{i}(x, z, P)=P_{i} a^{i j}(x) P_{j}+P_{i} b^{i} z-P_{i} f
$$

e

$$
\begin{aligned}
\bar{z} B(x, z, P) & =(|z|+k)(B(x, z, P)) \\
& =(|z|+k)\left(c^{i} P_{i}+d z-g\right) .
\end{aligned}
$$

Assim, como $\frac{\bar{z}}{k}=\frac{\bar{z}}{k}+1>1$ e $|\bar{z}|<|\bar{z}|^{2}$ pois $\bar{z}>1$, tem-se

$$
\begin{aligned}
\left|P_{i} b^{i} z-P_{i} f\right| & \leq\left|P_{i} b^{i}\right||\bar{z}|+\left|P_{i} f\right| \\
& \leq \frac{\lambda}{4}|P|^{2}+\frac{1}{\lambda}|b|^{2}|\bar{z}|^{2}+\frac{\lambda}{4}|P|^{2}+\frac{1}{\lambda}|f|^{2} \frac{|\bar{z}|^{2}}{k^{2}} \\
& \leq \frac{\lambda}{2}|P|^{2}+\frac{1}{\lambda}\left(|b|^{2}+k^{-2}|f|^{2}\right)|\bar{z}|^{2} \\
& \leq \frac{\lambda}{2}\left(|P|^{2}+2 \lambda^{-2}\left(|b|^{2}+k^{-2}|f|^{2}\right)|\bar{z}|^{2}\right) \\
& \leq \frac{\lambda}{2}\left(|P|^{2}+2 \bar{b}|\bar{z}|^{2}\right)
\end{aligned}
$$

de onde

$$
\text { (v) } \quad P_{i} b^{i} z-P_{i} f \geq-\frac{\lambda}{2}|P|^{2}-\frac{\lambda}{2}\left(2 \bar{b}|\bar{z}|^{2}\right) .
$$

De $(i v)$ e $(v)$ tem-se

$$
P_{i} A^{i}(x, z, P) \geq \frac{\lambda}{2}\left(|P|^{2}-2 \bar{b} \bar{z}^{2}\right)
$$

e para todo $\varepsilon$ tal que $0<\varepsilon<1$,

$$
\begin{aligned}
|\bar{z} B(x, z, P)| & =|\bar{z}|\left|c^{i} P_{i}+d z-g\right| \\
& \leq|\bar{z}||c||P|+|d||\bar{z}||z|+|\bar{z}||g| \\
& \leq \frac{\lambda}{2} \varepsilon|P|^{2}+\frac{1}{2 \varepsilon \lambda}|c|^{2}|\bar{z}|^{2}+|d||\bar{z}|^{2}+|\bar{z}|^{2}|g| \\
& \leq \frac{\lambda}{2}\left(\varepsilon|P|^{2}+\frac{1}{\varepsilon}\left(\lambda^{-2}|c|^{2}+\lambda^{-1}|d|+\lambda^{-1} k^{-1}|g|\right)|\bar{z}|^{2}\right)
\end{aligned}
$$

assim

$$
|\bar{z} B(x, z, P)| \leq \frac{\lambda}{2}\left(\varepsilon|P|^{2}+\frac{\bar{b}}{\varepsilon}|\bar{z}|^{2}\right) .
$$

Nosso primeiro resultado sobre limitações globais de soluções fracas é o seguinte teorema. 
Teorema 4.1 Se o operador satisfaz (4.1) e (4.2); $f \in L^{q}(\Omega), i=1, \ldots, n, g \in$ $L^{\frac{q}{2}}(\Omega)$ para algum $q>n ;$ e $u \in W^{1,2}(\Omega)$ é subsolução (supersolução) de $L u \leq g+D_{i} f(\geq)$ em $\Omega$ e satisfaz $u \leq 0(u \geq 0)$ em $\partial \Omega$, então tem-se

$$
\sup _{x \in \Omega} u(-u) \leq C\left(\left\|u^{+}\left(u^{-}\right)\right\|_{2}+k\right)
$$

onde $k=\lambda^{-1}\left(\|f\|_{q}+\|g\|_{\frac{q}{2}}\right)$ e $C:=C(n, \nu, q,|\Omega|)$.

Demonstração. Suponha que $u$ é subsolução de $L u \leq g+D_{i} f^{i}$, i.e,

$$
\int_{\Omega}\left(D_{i} v A^{i}(x, u, D u)-v B(x, u, D u)\right) d x \leq 0, \text { para todo } v \geq 0, v \in C_{0}^{1}(\Omega) .
$$

Para $\beta \geq 1, N>k$ define-se $H \in C^{1}(k,+\infty)$ por

$$
H(z)=\left\{\begin{array}{l}
z^{\beta}-k^{\beta} \quad z \in[k, N] \\
a z+b \quad z \geq N
\end{array}\right.
$$

Define $w=\bar{u}^{+}=u^{+}+k \quad\left(u^{+}=\max \{u, 0\}\right)$ e considere a função

$$
V=G(w)=\int_{k}^{w}\left|H^{\prime}(s)\right|^{2} d s .
$$

Tem-se que $V$ assim definido é maior ou igual a zero e $V \in C_{0}^{1}(\Omega)$ (consequência da regra da cadeia), assim de (4.2) e (4.1) tem-se

$$
\begin{aligned}
& \int_{\Omega} D_{i} v A^{i}(x, u, D u)-v B(x, u, D u) d x \\
& \quad=\int_{\Omega} G^{\prime}(w) D w A^{i}(x, u, D u)-G(w) B(x, u, D u) d x \leq 0,
\end{aligned}
$$

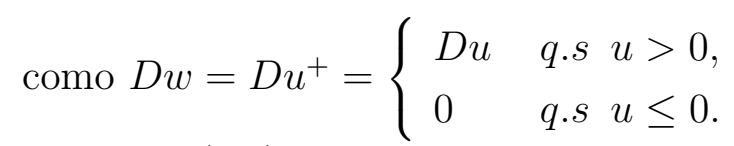

Por (4.9), tem-se

$$
\int_{\Omega} G^{\prime}(w) D w A^{i}(x, u, D u) d x \geq \frac{\lambda}{2} \int_{\Omega} G^{\prime}(w)|D w|^{2} d x-\frac{\lambda}{2} \int_{\Omega} 2 \bar{b} w^{2} G^{\prime}(w) d x .
$$

De (4.12), (4.13), (4.9), $G(s) \leq s G^{\prime}(s)$, e $D u=D w$, se $V=G(w)>0$ :

$$
\begin{aligned}
\int_{\Omega} G^{\prime}(w)|D w|^{2} d x & \leq \frac{2}{\lambda} \int_{\Omega} G(w) B(x, u, D u) d x+2 \int_{\Omega} \bar{b} w^{2} G^{\prime}(w) d x \\
& \leq \frac{2}{\lambda} \int_{\Omega} G^{\prime}(w) \frac{\lambda}{2} \varepsilon|D w|^{2}+\frac{\lambda}{2} \frac{\bar{b}}{\varepsilon} \frac{|G(w)|^{2}}{G^{\prime}(w)}+2 \int_{\Omega} \bar{b} w^{2} G^{\prime}(w) d x \\
& \leq \varepsilon \int_{\Omega} G^{\prime}(w)|D w|^{2}+\frac{\bar{b}}{\varepsilon} w^{2} G^{\prime}(w) d x+2 \int_{\Omega} \bar{b} w^{2} G^{\prime}(w) d x \\
& \leq \varepsilon \int_{\Omega} G^{\prime}(w)|D w|^{2}+\left(2+\frac{1}{\varepsilon}\right) \int_{\Omega} \bar{b} G^{\prime}(w) w^{2} d x
\end{aligned}
$$

Tomando $\varepsilon=\frac{1}{2}$, tem-se 


$$
\int_{\Omega} G^{\prime}(w)|D w|^{2} d x \leq 10 \int_{\Omega} \bar{b} G^{\prime}(w) w^{2} d x
$$

também de (4.11),

$$
G^{\prime}(w) D w=\left[H^{\prime}(w)\right]^{2} D w=D(H(w))
$$

assim

$$
\left\{\begin{aligned}
|D(H(w))|^{2} & =\left|H^{\prime}(w)\right|^{2}|D w|^{2} \\
& =G^{\prime}(w)|D w|^{2} .
\end{aligned}\right.
$$

Logo de (4.14) tem-se

$$
\int_{\Omega}|D H(w)|^{2} d x \leq 10 \int_{\Omega} \bar{b}\left|H^{\prime}(w)\right|^{2}|w|^{2} d x .
$$

Observe $H(w) \in W^{1,2}(\Omega)$. Pela Imersão de Sobolev temos que $W_{0}^{1,2}(\Omega)$ está imerso continuamente em $L^{\frac{2 n}{n-2}}(\Omega)$ se $n>2, W_{0}^{1,2}(\Omega)$ está imerso continuamente em $L^{q}(\Omega)$ para todo $q>1$ se $n=2$ e $W_{0}^{1,2}(\Omega)$ está imerso continuamente em $C^{0}(\bar{\Omega})$ se $n<2$.

Logo

$$
\begin{aligned}
|H(w)|_{L^{\frac{2 \hat{n}}{\hat{n}-2}}(\Omega)} & \leq C\left(\int_{\Omega} \bar{b}\left(H^{\prime}(w) w\right)^{2}\right)^{1 / 2} \\
& \leq C|\bar{b}|_{q / 2}^{1 / 2}\left|H^{\prime}(w) w\right|_{\frac{2 q}{q-2}}
\end{aligned}
$$

onde $\hat{n}=n$ se $n>2,2<\hat{2}<q, \quad C=C(n)$ se $n>2$ e $C=C(\hat{2},|\Omega|)$ se $n=2$. Assim

$$
|H(w)|_{L^{\frac{2 n}{n-2}(\Omega)}} \leq C\left|w H^{\prime}(w)\right|_{\frac{2 q}{q-2}}
$$

onde $C=C(n, \nu,|\Omega|)$. Observe que da definição de $H$ se $N \rightarrow \infty$ como $w H^{\prime}(w) \approx W^{\beta}$ a inclusão $w \in L^{2 \beta q / q-2}(\Omega)$ implica a inclusão mais forte $w \in L^{\frac{2 \hat{\beta} \hat{n}}{n}-2}$. Assim, se $q^{*}=\frac{2 q}{q-2}, \chi=\frac{\hat{n}(q-2)}{q(\hat{n}-2)}>1$ temos

$$
|w|_{\beta \chi q^{*}} \leq\left.(C \beta)^{1 / \beta}|| w\right|_{p q^{*}}
$$

Seja $\beta=\chi^{m}, m=0,1, \ldots$ Logo por (4.16) tem-se

$$
\begin{aligned}
& |w|_{\chi q^{*}} \leq|w|_{q^{*}} \\
& |w|_{\chi^{2} q^{*}} \leq(C \chi)^{\chi^{-1}}|w|_{\chi q^{*}} \leq C|C \chi|^{\chi^{-1}}|w|_{q^{*}} \\
& |w|_{\chi^{3} q^{*}} \leq\left(C \chi^{2}\right)^{\chi^{-2}}|w|_{\chi^{2} q^{*}} \leq C|C \chi|^{\chi^{-1}}\left|C \chi^{2}\right|^{\chi^{-2}}|w|_{q^{*}} \\
& |w|_{\chi^{N} q^{*}} \leq \Pi_{0}^{N-1}\left(C \chi^{m}\right)^{\chi^{-m}}\|w\|_{q^{*}} \\
& \leq C^{\sigma} \chi^{\tau}|w|_{q^{*}} \quad \text { (onde } \sigma=\sum_{0}^{N-1} \chi^{-m}, \tau=\sum_{0}^{N-1} m \chi^{-m} \text { ) } \\
& \leq C|w|_{q^{*}}
\end{aligned}
$$


onde $C=C(n, \nu, q,|\Omega|)$. Fazendo $N \rightarrow \infty$ tem-se

$$
\sup _{\Omega}|w| \leq C|| w \|_{q^{*}}, \quad q^{*}=\frac{2 q}{q-2},
$$

e como $q^{*} \geq 2 \Leftrightarrow \frac{2 q}{q-2} \geq 2$

$$
\sup _{\Omega}|w| \leq C|| w \|_{2}
$$

Assim (4.10) segue da definição de $w=u^{+}+k$ pois

$$
\sup _{\Omega}\left(u^{+}+k\right) \leq C\left\|u^{+}+k\right\|_{2} \Rightarrow \sup _{\Omega} u \leq \sup _{\Omega}\left(u^{+}+k\right) \leq C\left\|u^{+}+k\right\|_{2} .
$$

Se $u$ é supersolução, i.e,

$$
\int_{\Omega} D_{i} v A^{i}(x, u, D u)-v B(x, u, D u) d x \geq 0
$$

para todo $v \geq 0, v \in C_{0}^{1}(\Omega)$ da definição de $A^{i}, B$ tem-se que $-u$ é subsolução com $f^{i}=-f^{i}$ e $g=-g$ assim

$$
\begin{aligned}
\sup _{\Omega}(-u) \leq & C\left(\left\|(-u)^{+}\right\|_{2}+k\right) \quad\left((-u)^{+}=u^{-}\right) \\
& \leq C\left(\left\|u^{-}\right\|_{2}+k\right)
\end{aligned}
$$

Observação 4.4.1 A demonstração do Teorema 4.1 pode ser feita por escolha de outras funções testes. A iteração em cima usando $L^{p}$ normas foi introduzida por Moser (ver [13]).

Observação 4.4.2 Agora suponha que a hipótese $u \leq 0$ do Teorema $4.1 u$ generalizada a $u \leq l$ em $\partial \Omega$ para algum $\ell$ constante. Como

$$
\begin{aligned}
L(u-\ell) & =L u-L(l) \\
& =L u-\ell\left(D_{i} b^{i}+d\right),
\end{aligned}
$$

a conclusão do Teorema 4.1 vale para a função $u-l$ com $k$ substituindo por $\hat{k}=k+\lambda^{-1}\left(|l|\left(|| b\left\|_{q}+\right\| a \|_{q / 2}\right)\right)$ assim uma subsolução (supersolução) $u d e$ (4.3) satisfaz a estimativa

$$
\sup _{\Omega} u(-u) \leq C\left(\|u\|_{2}+\bar{k}+|l|\right),
$$

como antes $k=\lambda^{-1}\left(\|f\|_{q}+\|g\|_{q / 2}\right)$ e $C=C(n, \nu, q,|\Omega|)$. Em particular se $u$ é solução então (4.17) é valido para $|u|$, i.e,

$$
\sup _{\Omega}|u| \leq C\left(\|u\|_{2}+\bar{k}+|l|\right)
$$


Observação 4.4.3 A seguir, proporemos obter uma estimativa para $\sup _{\Omega} u$ independente de $\|u\|_{2}$ que é uma limitação a priori, o qual estende o principio do máximo fraco.

Teorema 4.2 Se o operador L satisfaz as condições (4.1), (4.2) e (4.4) e $f^{i} \in L^{q}(\Omega), i=1, \ldots, n, g \in L^{q / 2}(\Omega)$ para algum $q>n$, então se $u \in W^{1,2}(\Omega)$ é uma subsolução (supersolução) da equação (4.3), tem-se

$$
\sup _{\Omega} u(-u) \leq \sup _{\partial \Omega} u^{+}\left(u^{-}\right)+C k
$$

onde $k=\lambda^{-1}\left(\|f\|_{q}+\|g\|_{q / 2}\right)$ e $C=C(n, \nu, q,|\Omega|)$.

Demonstração. Suponha que $u$ é subsolução de $L u=g+D_{i} f^{i}$. Como $\int_{\Omega}\left(d v-b^{i} D_{i} v\right) d x \leq 0$ para todo $v \geq 0, v \in C_{0}^{1}(\Omega)$, temos que a constante $\ell=\sup _{\Omega} u^{+}$é supersolução. Assim, sem perda de generalidade, suponha $\ell=0$. Tem-se

$$
\int_{\Omega} a^{i j} D_{j} u D_{i} v-\left(b^{i}+c^{i}\right) v D_{i} u d x \leq \int_{\Omega} f^{i} D_{i} v-g v d x
$$

para todo $v \geq 0$ em $W_{0}^{1,2}(\Omega)$ com $u v \leq 0$.

Utilizaremos (4.8) com $b^{i}=d=0$ e $c$ substituído por $b+c$. Seja $k>0$, definimos $M=\sup _{\Omega} u^{+}$e escolhemos a função teste $v=\frac{u^{+}}{M+k+u} \in W_{0}^{1,2}(\Omega)$. Como

$$
\begin{aligned}
D_{i} v & =\frac{D_{i} u^{+}\left(M+k-u^{+}\right)+D_{i} u^{+}\left(u^{+}\right)}{\left(M+k-u^{+}\right)^{2}} \\
& =\left(D_{i} u^{+}\right) \frac{(M+k)}{\left(M+k-u^{+}\right)^{2}}
\end{aligned}
$$

tem-se,

$$
\begin{aligned}
a^{i j} D_{j} u D_{i} v & =a^{i j} D_{j} u D_{i} u \frac{(M+k)}{(M+k-u)^{2}} \\
& =a^{i j} D_{j} u^{+} D_{i} u^{+} \frac{(M+k)}{(M+k-u)^{2}} .
\end{aligned}
$$

Logo de (4.8) em (4.21) temos

$$
\begin{array}{r}
\frac{1}{2} \int_{\Omega} \frac{\left|D u^{+}\right|^{2}}{\left(M+k-u^{+}\right)^{2}} d x \leq \frac{1}{M+k} \int_{\Omega}\left[\frac{|b+c| u^{+}\left|D u^{+}\right|}{\left(M+k-u^{+}\right)}\right. \\
+\frac{u^{+}|g|}{\left(M+k-u^{+}\right)}+\frac{(M+k)|f|^{2}}{2 \lambda\left(M+k-u^{+}\right)^{2}} d x
\end{array}
$$

Como $k=\lambda^{-1}\left(\|f\|_{q}+\|g\|_{q / 2}\right)$ e $k<k+M-u^{+}$temos

$$
\frac{1}{k+M-u^{+}}<\frac{1}{k} \text { e } \frac{u^{+}}{M+k}<1 .
$$


Também tem-se

$$
\begin{aligned}
\frac{1}{M+k} \int_{\Omega} \frac{u^{+}|g|}{(M}+\frac{(M+k)|f|^{2}}{\left(k-u^{+}\right)}+\frac{1}{2 \lambda\left(M+k-u^{+}\right)^{2}} d x \\
\quad \leq \frac{1}{k} \int_{\Omega}|g| d x+\frac{1}{2 k^{2}} \int_{\Omega}|f|^{2} d x \\
\quad \leq \frac{1}{k} C_{1}\|g\|_{q / 2}+\frac{C_{2}^{2}}{2 k^{2}}\|f\|_{q}^{2} \\
\leq \frac{\lambda C_{1}\|g\|_{q / 2}}{\|f\|_{q}+\|g\|_{q / 2}}+\frac{\lambda^{2} C_{2}^{2}}{2\left(\|f\|_{q}+\|g\|_{q / 2}\right)}\|f\|^{2} \\
\leq \lambda \hat{C}(|\Omega|) .
\end{aligned}
$$

Assim de (4.23), (4.24) em (4.22) tem-se

$$
\int_{\Omega} \frac{D u^{+}}{\left(M+k-u^{+}\right)^{2}} d x \leq C+\frac{2}{\lambda} \int_{\Omega} \frac{|b+c|\left|D u^{+}\right|}{\left(M+k-u^{+}\right)} .
$$

Define $w=\log \left(\frac{M+k}{M+k-u}\right)$, temos

$$
D w=\frac{1}{M+k-u^{+}} D u^{+} \Longrightarrow|D w|^{2}=\frac{\left|D u^{+}\right|^{2}}{\left(M+k-u^{+}\right)^{2}} .
$$

Logo de (4.25) em (4.24) tem-se

$$
\begin{aligned}
\int_{\Omega}|D w|^{2} d x & \leq C\left(1+\frac{1}{\lambda} \int_{\Omega}|b+c||D w| d x\right) \\
& \leq C\left(1+\frac{1}{\lambda^{2}} \int_{\Omega}|b+c|^{2} d x\right)^{2}+\frac{1}{2} \int_{\Omega}|D w|^{2} d x
\end{aligned}
$$

Logo

$$
\int_{\Omega}|D w|^{2} d x \leq C\left(1+\lambda^{-2} \int_{\Omega}|b+c|^{2} d x\right) \leq C(\nu,|\Omega|)
$$

e pela Imersão de Sobolev,

$$
|w|_{2} \leq C(n, \nu,|\Omega|)
$$

Provaremos que $w$ é subsolução de uma equação da forma (4.3) $)_{1}$. Seja $\eta \in C_{0}^{1}(\Omega)$ satisfazendo $\eta \geq 0, \eta u \geq 0$ em $\Omega$. Substituindo em (4.21) a função teste $v=\frac{\eta}{M+k-u^{+}}$, tem-se

$$
\begin{gathered}
\int_{\Omega} a^{i j} D_{j} w D_{i} \eta+\eta a^{i j} D_{i} w D_{j} w-\left(b^{i}+c^{i}\right) \eta D_{i} w d x \\
\leq \int_{\Omega}-\frac{\eta g}{\left(M+k-u^{+}\right)}+\frac{\left(D_{i} \eta+\eta D_{i} w\right) f^{i}}{\left(M+k-u^{+}\right)} d x
\end{gathered}
$$


de onde

$$
\begin{aligned}
& \int_{\Omega} a^{i j} D_{j} w D_{i} \eta-\left(b^{i}+c^{i}\right) \eta D_{i} w+\lambda \eta \int_{\Omega}|D w|^{2} d x \\
& \quad \leq \int_{\Omega} \frac{|g| \eta}{k}+\frac{f^{i} D_{i} \eta}{\left(M+k-u^{+}\right)}+\frac{1}{2 \lambda k^{2}} \int_{\Omega}|f|^{2} \eta d x+\frac{\lambda}{2} \int_{\Omega} \eta|D w|^{2} d x \\
& \quad=\int_{\Omega}\left[\left(\frac{|g|}{k}+\frac{|f|^{2}}{2 \lambda k^{2}}\right) \eta+\frac{f^{i} D_{i} \eta}{\left(M+k-u^{+}\right)}\right] d x+\frac{\lambda}{2} \int_{\Omega} \eta|D w|^{2} d x
\end{aligned}
$$

Assim $\hat{g}=\frac{|g|}{k}+\frac{|f|^{2}}{2 \lambda k^{2}}, \hat{f}^{i}=\frac{f^{i}}{M+k-u^{+}} \mathrm{e}$

$$
\begin{aligned}
\|\hat{g}\|_{q / 2}^{q / 2} & =\int_{\Omega}\left(\frac{|g|}{k}+\frac{|f|^{2}}{2 \lambda k^{2}}\right)^{q / 2} d x \\
& \leq \int_{\Omega}\left(\frac{\lambda|g|}{|g|_{q / 2}}+\frac{\lambda}{2} \frac{|f|^{2}}{|f|_{q}}\right)^{q / 2} d x \\
& \leq \lambda^{q / 2}\left(1+\frac{1}{2}\right) .
\end{aligned}
$$

Então,

$$
\|\hat{g}\|_{q / 2} \leq 2 \lambda
$$

e analogamente

$$
\|\hat{f}\|_{q} \leq 1
$$

Pelo Teorema 4.1 aplicado a $w$ tem-se

$$
\begin{gathered}
\sup _{\Omega} w \leq C\left(1+\|w\|_{2}\right) \\
\leq C
\end{gathered}
$$

onde $C=C(n, \nu, q,|\Omega|)$, consequentemente

$$
\begin{aligned}
& \log \left(\frac{M+k}{M+k-u^{+}}\right) \leq C \\
\Longrightarrow & M+k \leq C(M+k)-C u^{+} \\
\Longrightarrow & u^{+} \leq(C-1)(n+k) \\
\Longrightarrow & u \leq \sup _{\partial \Omega} u^{+}+C k,
\end{aligned}
$$

$\log \mathrm{O}$

$$
\sup _{\Omega} u \leq \sup _{\partial \Omega}+C k
$$

ficando provado (4.20) para umr subsolução. O resultado para supersolução é obtido considerando $-u$ em lugar de $u$. 


\subsection{2}

\section{Propriedades Locais de Soluções Fracas}

Nesta seção concentraremos nas propriedades locais para soluções fracas.

Lembremos que, como na seção precedente,

$$
\begin{gathered}
P_{i} A^{i}(x, z, P) \geq \frac{\lambda}{2}|P|^{2}-\frac{1}{\lambda}\left(|b z|^{2}+|f|^{2}\right), \\
|B(x, z, P)| \leq|c||P|+|\lambda z|+|g|, \\
\bar{z}=|z|+k, \bar{b}=\lambda^{-2}\left(|b|^{2}+|c|^{2}+k^{-2}|f|^{2}\right)+\lambda^{-1}\left(|d|+k^{-1}|g|\right)
\end{gathered}
$$

para algum $k>0$, assim obtemos para todo $0<\varepsilon<1$

$$
\begin{aligned}
& P_{i} A^{i}(x, z, P) \geq \frac{\lambda}{2}\left(|P|^{2}-2 \bar{b} \bar{z}^{2}\right) \\
& |\bar{z} B(x, z, P)| \leq \frac{\lambda}{2}\left(\varepsilon|P|^{2}+\frac{\bar{b}}{\bar{\varepsilon}} \bar{z}^{2}\right) .
\end{aligned}
$$

Denotaremos por $\mathbf{a}(x)=\left[a^{i j}(x)\right]$, e adicionaremos a condição

$$
|A(x, z, P)| \leq|\mathbf{a}||P|+|b z|+|f| .
$$

Dividindo $L u=g+D_{i} f$ por $\frac{\lambda}{2}$ podemos assumir que $\lambda=2$, assim temse de (4.28), (4.30) e (4.31)

$$
\begin{aligned}
|A(x, z, P)| & \leq|\mathbf{a}||p|+|\mathbf{b}||z|+|f| \\
& \leq|\mathbf{a}||P|+2 \bar{b}^{1 / 2}\left|+2 k \bar{b}^{1 / 2}\right| z \mid \\
& \leq|\mathbf{a}||P|+2 \bar{b}^{1 / 2} \bar{z} \\
P A(x, z, P) & \geq|P|^{2}-2 \bar{b} \bar{z}^{2} \\
|\bar{z} B(x, z, P)| & \leq \varepsilon|P|^{2}+\frac{1}{\varepsilon} \bar{b} \bar{z}^{2}
\end{aligned}
$$

para todo $0<\varepsilon<1$.

Nesta seção definimos

$$
k=k(R)=\lambda^{-1}\left(R^{\sigma}\|f\|_{q}+R^{2 \sigma}\|g\|_{q / 2}\right)
$$

onde $R>0$ e $\sigma=1-\frac{n}{q}$.

Podemos estabelecer um resultado local similar ao Teorema 4.1.

Teorema 4.3 Seja o operador L satisfazendo (4.1) e (4.2) e suponha que $f^{i} \in L^{q}(\Omega), i=1, \ldots, n, g \in L^{q / 2}(\Omega)$ para algum $q>n$, então se $u \in W^{1,2}(\Omega)$ é subsolução (supersolução) de (4.3) em $\Omega$, temos para qualquer $B_{2 R}(y) \subset \Omega e$ $p>1$

$$
\sup _{B_{R}(y)}(-u) \leq C\left(R^{-m / p}\left\|u^{+}\left(u^{-}\right)\right\|_{L^{p}\left(B_{2 R}(y)\right)}+k(R)\right) .
$$


Demonstração. Suponha que $R=1$ e $k>0$. O caso geral é obtido através da transformação $x \mapsto \frac{x}{R}$ e fazendo $k \rightarrow 0$. Define para $\beta \neq 0$ e $\eta \in C_{0}^{1}\left(B_{4}\right)$ a função teste $v=\eta^{2} \bar{u}^{\beta}$ onde $\bar{u}=u+k$. Então $v$ pode ser considerada uma função teste em

$$
\int_{\Omega}\left(D_{i} v A^{i}(x, u, D u)-v B(x, u, D u)\right) d x \leq 0(\geq 0) .
$$

Como

$$
D v=2 \eta D \eta \bar{u}^{\beta}+\beta \eta^{2} \bar{u}^{\beta-1} D u,
$$

substituindo (4.35) em (4.34) tem-se

$$
\begin{aligned}
\beta \int_{\Omega} \eta^{2} \bar{u}^{\beta-1} D & \cdot A(x, u, D u) d x+2 \int_{\Omega} \eta D \eta \cdot A(x, u, D u) \bar{u}^{\beta} d x \\
- & \int_{\Omega} \eta^{2} \bar{u}^{\beta} B(x, u, D u) d x \\
\leq 0 & \text { se } u \text { é subsolução } \\
\geq 0 & \text { se } u \text { é supersolução. }
\end{aligned}
$$

Usando (4.32) temos que

$$
D u \cdot A(x, u, D u) \geq|D u|^{2}-2 \bar{b} \bar{u}^{2}
$$

e multiplicando esta ultima desigualdade por $\eta^{2} \bar{u}^{\beta-1}$ tem-se para qualquer $0<\varepsilon<1$,

$$
\left\{\begin{aligned}
& \eta^{2} \bar{u}^{\beta-1} D u \cdot A(x, u, D u) \geq \eta^{2} \bar{u}^{\beta-1}|D u|^{2}-2 \bar{b} \eta^{2} \bar{u}^{\beta+1}, \text { também } \\
&\left|\eta D \eta \cdot A(x, u, D u) \bar{u}^{\beta}\right| \leq \eta|D \eta||\mathbf{a}||D u| \bar{u}^{\beta}+2 \bar{b}^{1 / 2} \bar{u}|\eta||D \eta| \bar{u}^{\beta} \\
& \leq|\mathbf{a}| \eta|D \eta|\left|\bar{u}^{\beta}\right||D u|+2 \bar{b}^{1 / 2} \eta|D \eta| \bar{u}^{\beta+1} \\
& \leq \frac{\varepsilon}{2} \eta^{2} \bar{u}^{\beta-1}|D u|^{2}+\left(1+\frac{|\mathbf{a}|^{2}}{2 \varepsilon}\right)|D \eta|^{2} \bar{u}^{\beta+1}+\bar{b} \eta^{2} \bar{u}^{\beta+1} \\
&\left|\eta^{2} \bar{u}^{\beta} B(x, u, D u)\right|=\eta^{2} \bar{u}^{\beta-1} \bar{u}(x, u, D u) \\
& \leq \eta^{2} \bar{u}^{\beta-1}\left(\varepsilon|D u|^{2}+\frac{1}{\varepsilon} \bar{b} \eta^{2} \bar{u}^{2}\right) \\
& \leq \varepsilon \eta^{2} \bar{u}^{\beta-1}|D u|^{2}+\frac{1}{\varepsilon} \bar{b} \eta^{2} \bar{u}^{\beta+1}
\end{aligned}\right.
$$

Assumindo que $\beta>0$ se $u$ é uma subsolução e $\beta<0$ se $u$ é supersolução, e 
escolhendo $\varepsilon+\min \left\{\frac{1}{2}, \frac{|\beta|}{4}\right\}$ tem-se por (4.36) e (4.37) que

$$
\begin{aligned}
\int_{\Omega} \eta^{2} \bar{u}^{\beta-1} & |D u|^{2} d x \\
& \leq \int_{\Omega} \eta^{2} \bar{u}^{\beta-1} D u \cdot A(x, u, D u)+2 \bar{b} \eta^{2} \bar{u}^{\beta-1} d x \\
& =\frac{1}{\beta} \int_{\Omega} 2 \eta D \eta A(x, u, D u) \bar{u}^{\beta} d x+\int_{\Omega} \eta^{2} \bar{u}^{2} B(x, u, D u) d x+2 \int_{\Omega} \bar{b} \eta^{2} \bar{u}^{\beta+1} d x \\
& \leq \frac{2}{\beta} \int_{\Omega} \frac{\varepsilon}{2} \eta^{2} \bar{u}^{\beta-1}|D u|^{2} d x+\int_{\Omega}\left(1+\frac{|\mathbf{a}|^{2}}{2|\varepsilon|}\right)|D \eta|^{2} \bar{u}^{\beta-1}+\varepsilon \eta^{2} \bar{u}^{\beta-1}|D u|^{2}+\frac{1}{\varepsilon} \bar{b} \eta^{2} \bar{u}^{\beta+1} d x \\
& \leq \frac{1}{4} \int_{\Omega} \eta^{2} \bar{u}^{\beta-1}|D u|^{2} d x+\frac{1}{2} \int_{\Omega} \eta^{2} \bar{u}^{\beta-1}|D u|^{2} d x+\int_{\Omega}\left[\bar{b} \eta^{2}+\left(1+|\mathbf{a}|^{2}\right)|D \eta|^{2}\right] \bar{u}^{\beta+1} d x
\end{aligned}
$$

de onde,

$$
\int_{\Omega} \eta^{2} \bar{u}^{\beta-1}|D u|^{2} d x \leq C(|\beta|) \int_{\Omega}\left(\bar{b} \eta^{2}+\left(1+|\mathbf{a}|^{2}\right)|D \eta|^{2}\right)|\bar{u}|^{\beta+1} d x
$$

em que $C(|\beta|)$ é limitado se $|\beta|$ é limitado longe de zero. Introduzindo a função

$$
w= \begin{cases}\bar{u}^{\frac{\beta+1}{2}} & \text { se } \beta \neq-1 \\ \log \bar{u} & \text { se } \beta=-1\end{cases}
$$

se $\gamma=\beta+1$, tem-se

$$
D w= \begin{cases}\frac{\beta+1}{2} \bar{u}^{\frac{\beta-1}{2}} D \bar{u} & \text { se } \beta \neq-1 \\ \frac{1}{\bar{u}} D \bar{u} & \text { se } \beta=-1\end{cases}
$$

Logo de (4.37) tem-se

$$
\int_{\Omega} \eta^{2}|D w|^{2} d x \leq \begin{cases}\frac{\gamma^{2}}{4} C(|\beta|) \int_{\Omega}\left(\bar{b} \eta^{2}+\left(1+|\mathbf{a}|^{2}\right)|D \eta|^{2}\right)|w|^{2} d x & \text { se } \beta \neq-1 \\ C(|\beta|) \int_{\Omega}\left(\bar{b} \eta^{2}+\left(1+|\mathbf{a}|^{2}\right)|D \eta|^{2}\right) d x & \text { se } \beta=-1 .\end{cases}
$$

O processo de iteração desejado pode ser obtido da primeira parte de (4.38).

Pelo teorema de imersão de Sobolev

$$
W^{1, p}(\Omega) \subset \begin{cases}L^{\frac{n p}{n-p}}(\Omega) & \text { se } p<n \\ C^{0}(\Omega) & \text { se } p>n,\end{cases}
$$

e existe $C=C(n, p)$ tal que

$$
\begin{cases}|u|_{\frac{n p}{n-p}} \leq C|D u|_{p} & \text { para } p<n \\ \sup _{\Omega}|u| \leq C|\Omega|^{\frac{1}{n}-\frac{1}{p}}|D u|_{p} & \text { para } p>n\end{cases}
$$

Podemos deduzir que 


$$
|\eta w|_{\frac{2 \hat{n}}{\hat{n}-2}}^{2} \leq C \int_{\Omega}|\eta D w|^{2}+|w D \eta|^{2} d x
$$

onde $\hat{n}=n$ se $n>2,2<\hat{2}<q$ e $C=C(\hat{n})$. Usando a desigualdade de Hölder, e a desigualdade de interpolação para espaços $L^{p}$ tem-se,

$$
\begin{aligned}
\int_{\Omega} \bar{b}(\eta w)^{2} d x & \leq|\bar{b}|_{q / 2}|\eta w|_{\frac{2 q}{q-2}}^{2} \\
& \leq|\bar{b}|_{q / 2}\left(\varepsilon\|\eta w\|_{\frac{2 \hat{n}}{\hat{n}-2}}+\varepsilon^{-\sigma}\|\eta w\|_{2}\right)^{2}
\end{aligned}
$$

em que $\sigma=\frac{\hat{n}}{q-\hat{n}}$.

Logo de (4.40), (4.41) e (4.39) para $\varepsilon$ tal que $|\bar{b}|_{q / 2} \varepsilon=\frac{1}{2}$, tem-se

$$
|\eta w|_{\frac{2 \hat{n}}{\hat{n}-2}} \leq C(1+|\gamma|)^{\sigma+1}\|(\eta+|D \eta|) w\|_{2}
$$

onde $C=C(\hat{n}, \Lambda, \nu, q,|B|)$ é limitado se $|\beta|$ é limitado longe de zero.

Agora vamos especificar a função de corte $\eta$. Sejam $r_{1}, r_{2}$ tais que $1 \leq r_{1}<r_{2} \leq 3$ e seja

$$
\eta= \begin{cases}1 & \text { em } B_{r_{1}} \operatorname{com}|D \eta| \leq \frac{2}{r_{2}-r_{1}} \\ 0 & \text { em } \Omega \backslash B_{r_{2}}\end{cases}
$$

Se $\chi=\frac{\hat{n}}{\hat{n}-2}$ tem-se de $(4.42)$

$$
\begin{aligned}
\|w\|_{L^{2 \chi}\left(B_{r_{1}}\right)} & \leq C(1+|\gamma|)^{\sigma+1}\|(\eta+|D \eta|) w\|_{2} \\
& \leq C(1+|\gamma|)^{\sigma+1}\left\|\left(1+\frac{2}{r_{2}-r_{1}}\right) w\right\|_{L^{2}\left(B_{r_{2}}\right)} \\
& =\frac{C(1+|\gamma|)^{\sigma+1}}{r_{2}-r_{1}}\|w\|_{L^{2}\left(B_{r_{2}}\right)} .
\end{aligned}
$$

Para $r<4$ e $p \neq 0$, introduzimos

$$
\phi(p, r)=\left(\int_{B_{r}}|\bar{u}|^{p} d x\right)^{1 / p}
$$

Tem-se pela proposição 2.0.2 que $\phi(\infty, r)=\lim _{p \rightarrow \infty} \phi(p, r)=\sup _{\Omega} \bar{u}$, e

$$
\phi(-\infty, r)=\lim _{p \rightarrow-\infty} \phi(p, r)=\inf _{B_{r}} \bar{u}
$$

De (4.43) e a definição de $w$ tem-se

$$
\left(\int_{B_{r}}|\bar{u}|^{\chi \gamma} d x\right)^{\frac{1}{2 \chi}} \leq \frac{C(1+|\gamma|)^{\sigma+1}}{r_{2}-r_{1}}\left(\int_{B_{r_{2}}}|\bar{u}|^{\gamma} d x\right)^{1 / 2}
$$


de onde, elevando a $\frac{2}{|\gamma|}$ tem-se,

$$
\left(\int_{B_{r_{1}}}|\bar{u}|^{\chi \gamma} d x\right)^{\frac{1}{\chi \gamma}} \leq\left(\frac{C(1+|\gamma|)^{\sigma+1}}{r_{2}-r_{1}}\right)^{2 /|\gamma|}\left(\int_{B_{r_{2}}}|\bar{u}|^{\gamma} d x\right)^{1 / \gamma}
$$

isto é,

$$
\begin{aligned}
& \phi\left(\chi \gamma, r_{1}\right) \leq\left(\frac{C(1+|\gamma|)^{\sigma+1}}{r_{2}-r_{1}}\right)^{2 /|\gamma|} \phi\left(\gamma, r_{2}\right) \text { se } \gamma>0 \\
& \phi\left(\gamma, r_{2}\right) \leq\left(\frac{C(1+|\gamma|)^{\sigma+1}}{r_{2}-r_{1}}\right)^{2 /|\gamma|} \phi\left(\chi \gamma, r_{1}\right) \text { se } \gamma<0
\end{aligned}
$$

Agora podemos iterar estas desigualdades, por exemplo se $u$ é subsolução temse $\beta>0$ e $\gamma=\beta+1>1 \operatorname{logo}$ tomando $\beta>1$ colocamos $\gamma=\gamma_{m}=\chi^{m} p$ e $r_{m}=1+2^{-m}, m=0,1,2, \ldots$ Por $(4.44)$

$$
\begin{aligned}
\phi\left(\chi^{m+1} p, r_{m+1}\right) & \leq\left(\frac{C\left(1+\left(\chi^{m} p\right)^{\sigma+1}\right)}{2^{-(m+1)}}\right)^{\frac{2}{\chi^{m} p}} \phi\left(\chi^{m} p, r_{m}\right) \\
& \leq \Pi_{0}^{m}\left(C 2^{i+1}\left(1+\chi^{j} p\right)^{\sigma+1}\right)^{2 \chi^{-j} p} \phi(p, 2) \\
& \leq \Pi_{j=0}^{m}\left(C 2^{j+2}\left(\chi^{j} p\right)^{\sigma+1}\right)^{2 \chi^{-j} p} \phi(p, 2) \\
& \leq \Pi_{j=0}^{m}\left[(\hat{C} \chi)^{j}\right]^{2(\sigma+1) \chi^{-j} p} \phi(p, 2) \\
& \leq(\hat{C} \chi)^{2(\sigma+1) p \sum_{j=0}^{m} j \chi^{-j}} \phi(p, 2) \\
& =\hat{C} \phi(p, 2)
\end{aligned}
$$

em que $\hat{C}=\hat{C}(\hat{n}, \Lambda, \nu, q, p)$.

Tomando limite quando $m \rightarrow \infty$ tem-se

$$
\sup _{B_{1}} \bar{u} \leq C|| \bar{u} \|_{L^{p}\left(B_{2}\right)}
$$

usando a transformação $x \mapsto \frac{x}{R}$, tem-se

$$
\begin{aligned}
\sup _{B_{R}} \bar{u} & =\sup _{B_{1}} \bar{u}\left(\frac{x}{R}\right) \\
& \leq C \| \bar{u}\left(\frac{x}{R}\right)||_{L^{p}\left(B_{2}\right)} \\
& =C\left(\int_{B_{2}}\left|\bar{u}\left(\frac{x}{R}\right)\right|^{p} d x\right)^{1 / p} \\
& =C\left(R^{-n} \int_{B_{R}}|\bar{u}|^{p} d x\right)^{1 / p} \\
& =C R^{-n / p}|\bar{u}|_{L^{P}\left(B_{2 R}\right)} .
\end{aligned}
$$


Logo como $\bar{u}=u+k$ tem-se

$$
\begin{aligned}
\sup _{B_{R}} u+k & \leq C R^{-n / p}|u+k|_{L^{p}\left(B_{2 R}\right)} \\
& \leq C R^{-n / p}\left|u^{+}+k\right|_{L^{p}\left(B_{2 R}\right)} \\
& \leq C\left(R^{-n / p}|| u^{+} \|_{L^{p}\left(B_{2 R}\right)}+k(R)\right) .
\end{aligned}
$$

de onde $\sup _{B_{R}} u \leq C\left(R^{-n / p}\left\|u^{+}\right\|_{L^{P}\left(B_{2 R}\right)}+k(R)\right)$.

Está provado (4.33), isto é, o Teorema 4.3 fica provado.

Um resultado importante no desenvolvimento das propriedades locais de soluções fracas será a seguinte Desigualdade de Harnack para supersoluções.

Teorema 4.4 Se o operador $L$ satisfaz (4.1), (4.2) e $f^{i} \in L^{q}(\Omega), g \in L^{q / 2}(\Omega)$ para algum $q>n$, então se $u \in W^{1,2}(\Omega)$ é uma supersolução de (4.3) em $\Omega$ não negativa em uma bola $B_{4 R}(y) \subset \Omega$ e $1 \leq p<\frac{n}{n-2}$, temos

$$
-R^{-m / p}\|u\|_{L^{p}\left(B_{2 R}(y)\right)} \leq C\left(\inf _{B_{R}(y)} u+k(R)\right)
$$

onde $C=C\left(n, \frac{\Lambda}{\lambda}, \nu R, q, p\right)$.

Demonstração. Para quaisquer $p, p_{0}$ tal que $0<p_{0}<p<\chi=\frac{\hat{n}}{\hat{n}-2}$ tem-se

$$
\begin{aligned}
& \phi(p, 2) \leq C \phi\left(p_{0}, 3\right) \\
& \phi\left(-p_{0}, 3\right) \leq C \phi(-\infty, 1),
\end{aligned}
$$

$\operatorname{com} C=C\left(\hat{n}, \Lambda, q, p, p_{0}\right)$, pois

$$
\begin{aligned}
\phi(p, 2) & =\left(\int_{B_{2}}|\bar{u}|^{p}\right)^{1 / p} \leq \sup _{B_{2}} \bar{u} \cdot\left|B_{2}\right|^{1 / p} \\
& \leq C|\bar{u}|_{L^{p_{0}\left(B_{3}\right)}}\left|B_{2}\right|^{1 / p} \leq C \phi\left(p_{0}, 3\right) .
\end{aligned}
$$

Também,

$$
\begin{aligned}
\phi\left(-p_{0}, 3\right) & =\left(\int_{B_{3}}|\bar{u}|^{-p_{0}} d x\right)^{-\frac{1}{p_{0}}} \\
& \leq\left(\int_{B_{3}}\left(\inf _{B_{3}} \bar{u}\right)^{-p_{0}} d x\right)^{-\frac{1}{p_{0}}} \\
& \leq\left|B_{3}\right|^{-\frac{1}{p_{0}}} \inf _{B_{3}} \bar{u} \\
& \leq\left|B_{3}\right|^{-\frac{1}{p_{0}}} \inf _{B_{1}} \bar{u} \\
& \leq C \phi(-\infty, 1),
\end{aligned}
$$

assim a conclusão do Teorema 4.4 seguira se podemos demonstrar que para algum $p_{0}>0$

$$
\phi\left(p_{0}, 3\right) \leq C \phi\left(-p_{0}, 3\right)
$$


Para provar isto, se $B_{2 r}$ é qualquer bola de raio $2 r$ em $B_{4}=B_{4}(y)$ escolhemos uma função de corte $\eta$ tal que

$$
\eta=\left\{\begin{array}{lll}
1 & \text { em } B_{r} \quad \text { e }|D \eta| \leq \frac{2}{r} \\
0 & \text { em } B_{r} \backslash B_{4}
\end{array}\right.
$$

De $(4.38)_{2}$ usando a desigualdade de Hölder, tem-se

$$
\begin{aligned}
\int_{B_{r}}|D w| d x & \leq C r^{n / 2}\left(\int_{B_{r}}|D w|^{2} d x\right)^{1 / 2} \\
& \leq C r^{n / 2}\left(C\left(1+\left(\frac{2}{r}\right)^{2}\right)\right)^{1 / 2} \pi 2^{n} \\
& =C r^{n / 2}\left(1+\frac{1}{r}\right) \\
& \leq C r^{n-1}
\end{aligned}
$$

Pelo Teorema 2.15 existe $p_{0}>0$ dependendo de $n, \Lambda$ e $\nu$ tal que para $w_{0}=\frac{1}{\mid B_{3}} \mid \int_{B_{3}} w d x$ tem-se

$$
\int_{B_{3}} e^{p_{0}\left|w-w_{0}\right|} d x \leq C(n, \Lambda, \nu)
$$

e assim

$$
\int_{B_{3}} e^{p_{0} w} d x \int_{B_{3}} e^{-p_{0} w} d x^{\prime} \leq C e^{p_{0} w_{0}} e^{-p_{0} w_{0}}=C
$$

Logo, como $e^{p_{0} w}=e^{p_{0}|\log \bar{u}|}=\bar{u}^{p_{0}}$, tem-se

$$
\int_{B_{3}}|\bar{u}|^{p_{0}} d x \int_{B_{3}}|\bar{u}|^{-p_{0}} d x^{\prime} \leq C
$$

de onde,

$$
\left(\int_{B_{3}}|\bar{u}|^{p_{0}} d x\right)^{1 / p_{0}}\left(\int_{B_{3}}|\bar{u}|^{-p_{0}} d x^{\prime}\right)^{1 / p_{0}} \leq C^{1 / p_{0}}=C .
$$

Logo,

$$
\begin{aligned}
& \left(\int_{B_{3}}|\bar{u}|^{p_{0}} d x\right)^{1 / p_{0}} \leq C\left(\int_{B_{3}}|\bar{u}|^{-p_{0}} d x^{\prime}\right)^{-1 / p_{0}} \\
\Longrightarrow & \phi\left(p_{0}, 3\right) \leq C \phi\left(-p_{0}, 3\right),
\end{aligned}
$$

ficando provado (4.47). Assim de (4.46) e (4.47) tem-se

$$
R^{-n / p}|| \bar{u} \|_{L^{p}\left(B_{2 R}\right)} \leq C\left(\inf _{B_{R}} \bar{u}\right)
$$

Como $\bar{u}=u+k$ tem-se,

$$
R^{-n / p}\|u\|_{L^{p}\left(B_{2 R}\right)} \leq C\left(\inf _{B_{R}} u+k(R)\right),
$$


onde $C=C(n, \Lambda / \lambda, \nu R, q, p)$, ficando provado o Teorema 4.4 .

Como consequência da Desigualdade de Harnack Fraca serão provados o Princípio do Máximo Forte, a Desigualdade de Harnack e a Propriedade de Continuidade de Holder.

\subsection{3}

\section{Principio do Máximo Forte}

Teorema 4.5 Seja o operador L satisfazendo (4.1), (4.2) e (4.4) e seja $u \in W^{1,2}(\Omega)$ satisfazendo $L u \geq 0$ em $\Omega$. Então se em alguma bola $B \Subset \Omega$ tem-se

$$
\sup _{B} u=\sup _{\Omega} u \geq 0
$$

a função u deve ser constante em $\Omega$ e a igualdade vale em (4.4), isto é,

$$
\int_{\Omega} d v-b^{i} D_{i} v d x=0 \text { para todo } v \geq 0, v \in C_{0}(\Omega),
$$

quando $u \neq 0$.

Demonstração. Escrevendo $B=B_{R}(y)$ sem perda de generalidade assumimos que $B_{4 R}(y) \subset \Omega$.

Seja $M=\sup _{\Omega} u$ e aplicamos a desigualdade de Harnack com $f=0, q=0$ e com $p=1$ a supersolução $v=M-u$, tem-se

$$
R^{-n} \int_{B_{2 R}}(M-u) d x \leq C \inf _{B_{R}(y)}(M-u)+\underbrace{k(R)}_{0}
$$

pois

$$
k(R)=\lambda^{-1}(R^{\sigma} \underbrace{\|f\|_{q}}_{0}+R^{2 \sigma} \underbrace{\|g\|_{q / 2}}_{0})=0 .
$$

Portanto $M=\sup _{\Omega} u=\sup _{B} u \Longrightarrow \inf _{B}(M-u)=0$, logo de (4.49) podemos deduzir $M=u$ em $B_{2 R}$, (como $M-u \geq 0$ em $\left.B_{2 R}\right)$. Como $\sup _{\Omega} u=\sup _{B_{R}} u=M$, então $u=M$ em $B_{2 R}(y)$ e

$$
B_{4 R}(y) \subset \Omega \Longrightarrow \sup _{\Omega} u=\sup _{B_{2 R}} u=M \Longrightarrow u=M \operatorname{em~} B_{4 R}(y) .
$$

Dado $\bar{y} \in \Omega$ como $\Omega$ é um domínio conexo, existe um numero finito de bolas de raio $r_{i}<R$ tal que a curva que une $\bar{y}$ e $y$ esta contida em $\Omega$ e

$$
\mathcal{C} \subset B_{r_{i}}\left(y_{i}\right) \cup \ldots \cup B_{r_{n}}\left(y_{n}\right) \subset \Omega
$$

e pelo visto acima $u=M$ em $B_{r_{i}}\left(y_{i}\right)$. Assim $u=M$ em $\bar{y}$, logo como $\bar{y}$ é arbitrário, temos $u=M$ em $\Omega$. 


\subsection{4}

\section{Desigualdade de Harnack}

Teorema 4.6 Se o operador L satisfaz as condições (4.1) e (4.2) e u $\in$ $W^{1,2}(\Omega)$ satisfaz $u \geq 0$ em $\Omega$ e $L u=0$ em $\Omega$ então $\forall B_{4 R}(y) \subset \Omega$ tem-se

$$
\sup _{B_{R}(y)} u \leq C \inf _{B_{R}(y)} u
$$

onde $C=C(n, \Lambda / \lambda, \nu R)$.

Demonstração. Seguindo como na demonstração do Teorema $4.4 \mathrm{com}$ $f=0, g=0, p=1$ tem-se que

$$
k=k(R)=\lambda^{-1}(R^{\sigma} \underbrace{\|f\|_{q}}_{0}+R^{2 \sigma} \underbrace{\|g\|_{q / 2}}_{0})=0 .
$$

Assim de (4.33) com $u \geq 0$ (i.e. $u^{+}=u$ )

$$
\begin{aligned}
\sup _{B_{R}(y)} u & \leq C\left(R^{-n}\|u\|_{L^{p}\left(B_{2 R}(y)\right)}\right) \\
& \leq C \inf _{B_{R}(y)} u .
\end{aligned}
$$

Corolário 4.7 Se L e u satisfazem as hipóteses do Teorema 4.6 então para todo $\Omega^{\prime} \Subset \Omega$ tem-se

$$
\sup _{\Omega^{\prime}} u \leq C \inf _{\Omega^{\prime}} u
$$

onde $C=C\left(n, \Lambda / \lambda, \nu, \Omega^{\prime}, \Omega\right)$.

\subsection{5}

\section{Propriedades de Hölder}

Nesta seção apresentaremos as propriedades de continuidade de Holder para as soluções de (4-3). Estes resultados são fundamentais para a teoria das equações quase-lineares de segunda ordem e foram descobertos por De Giorgi para operadores da forma $L u=D_{i}\left(a^{i j}(x) D_{j} u\right)$. Esta descoberta iniciou a teoria das equações quase-lineares em mais de duas variáveis.

Teorema 4.8 Se o operador L satisfaz as condições (4.1) e (4.2) e $f^{i} \in L^{q}(\Omega)$, $g \in L^{q / 2}(\Omega)$ para algum $q>n$ então se $u \in W^{1,2}(\Omega)$ é solução de $L u=g+D_{i} f^{i}$ em $\Omega$, segue-se que u é localmente Hölder contínua em $\Omega$ e para toda bola 
$B_{0}=B_{R_{0}}(y) \subset \Omega$ e $R \leq R_{0}$ tem-se

$$
\operatorname{osc}_{B_{R}(y)} u \leq C R^{\alpha}\left(R_{0}^{\alpha} \sup _{B_{0}}|u|+k\right),
$$

onde $C=C\left(n, \Lambda / \lambda, \nu, q, R_{0}\right)$ e $\alpha=\alpha\left(n, \Lambda / \lambda, \nu R_{0}, q\right)$ são constantes positivas e $k=\lambda^{-1}\left(\|f\|_{q}+\|g\|_{q / 2}\right)$.

Demonstração.

Sem perda de generalidade $R \leq \frac{R_{0}}{4}$. Denotamos

$$
M_{0}=\sup _{B_{0}}|u|, M_{4}=\sup _{B_{4 R}} u, m_{4}=\inf _{B_{4 R}} u, M_{1}=\sup _{B_{R}} u, m_{1}=\inf _{B_{R}} u .
$$

Então tem-se

$$
\begin{aligned}
& L\left(M_{4}-u\right)=M_{4}\left(D_{i} b^{i}+d\right)-D_{i} f^{i}-g \\
& L\left(M-m_{4}\right)=-m_{4}\left(D_{i} b^{i}+d\right)+D_{i} f^{i}+g .
\end{aligned}
$$

Considerando,

$$
\begin{aligned}
& \bar{k}(R)=\lambda^{-1} R^{\sigma}\left(\|f\|_{q}+M_{0}\|b\|_{q}\right)+\lambda^{-1} R^{2 \sigma}\left(\|g\|_{q / 2}+M_{0}\|d\|_{q / 2}\right), \\
& \sigma=1-\frac{n}{q},
\end{aligned}
$$

e aplicando a desigualdade de Harnack (4.45) com $p=1$ às funções $M_{4}-u, u-$ $m_{4}$ em $B_{4 R}$ temos

$$
\begin{aligned}
& R^{-n} \int_{B_{2 R}}\left(M_{4}-u\right) d x \leq C\left(M_{4}-M_{1}+\bar{k}(R)\right) \\
& R^{-n} \int_{B_{2 R}}\left(u-m_{4}\right) d x \leq C\left(m_{1}-m_{4}+\bar{k}(R)\right) .
\end{aligned}
$$

Logo somando (4.50) e (4.51) tem-se

$$
\begin{aligned}
R^{-n} \int_{B_{2 R}}\left(M_{4}-u\right) d x+ & R^{-n} \int_{B_{2 R}}\left(u-m_{4}\right) d x \\
& =R^{-n} \int_{B_{2 R}} M_{4}-m_{4} d x \\
& =R^{-n}\left(M_{4}-m_{4}\right)\left|B_{2 R}\right| \\
& =R^{-n}\left(M_{4}-m_{4}\right) C(2 R)^{n} \\
& =C_{n}\left(M_{4}-m_{4}\right) \\
& \leq C_{n}\left(M_{4}-m_{4}+m_{1}-M_{1}+\bar{k}(R)\right)
\end{aligned}
$$

de onde

$$
M_{4}-m_{4} \leq C\left(M_{4}-m_{4}+m_{1}-M_{1}+\bar{k}(R)\right),
$$


assim escrevendo $w(R)=$ osc $u=M_{1}-m_{1}$, temos de (4.52)

$$
w(R) \leq \gamma w(4 R)+\bar{k}(R)
$$

Provaremos o seguinte Lema:

Lema 4.4.1 Seja w uma função não decrescente sobre o intervalo $\left(0, R_{0}\right)$ satisfazendo para todo $R \leq R_{0}$, a desigualdade

$$
w(\tau R) \leq \gamma w(R)+\sigma(R)
$$

onde $\sigma$ é também não decrescente e $0<\gamma, \tau<1$. Então para todo $\mu \in(0,1)$ e $R \leq R_{0}$ tem-se

$$
w(R) \leq C\left(\left(\frac{R}{R_{0}}\right)^{\alpha} w\left(R_{0}\right)+\sigma\left(R^{\mu} R_{0}^{1-\mu}\right)\right),
$$

onde $C=C(\gamma, \tau)$ e $\alpha=\alpha(\gamma, \tau, \mu)$ são constantes positivas.

Demonstração. Fixemos $R_{1} \leq R_{0}$, logo para todo $R \leq R_{0}$ por (4.53), tem-se

$$
\begin{aligned}
w(\tau R) \leq & \gamma w(R)+\sigma(R) \\
& \leq \gamma w(R)+\sigma\left(R_{1}\right)
\end{aligned}
$$

Logo,

$$
\begin{aligned}
w\left(\tau^{2} R_{1}\right) & \leq w\left(\tau\left(\tau R_{1}\right)\right) \\
& \leq \gamma w\left(\tau R_{1}\right)+\gamma\left(\tau R_{1}\right) \\
& \leq \gamma\left(w\left(R_{1}\right)\right)+\gamma\left(R_{1}\right)+\gamma\left(R_{1}\right) \\
& =\gamma^{2} w\left(R_{1}\right)+\gamma\left(R_{1}\right)(1+\gamma) .
\end{aligned}
$$

Analogamente,

$$
\begin{aligned}
w\left(\tau^{3} R_{1}\right) & =w\left(\tau\left(\tau^{2} R_{1}\right)\right) \leq \gamma w\left(\tau^{2} R_{1}\right)+\gamma\left(\tau^{2} R_{1}\right) \\
& \leq \gamma\left(\gamma^{2} w\left(R_{1}\right)+\gamma\left(R_{1}\right)(1+\gamma)\right)+\gamma\left(R_{1}\right) \\
& \leq \gamma^{3} w\left(R_{1}\right)+\gamma\left(R_{1}\right)\left(1+\gamma+\gamma^{2}\right) .
\end{aligned}
$$

Assim sucessivamente

$$
\begin{aligned}
w\left(\tau^{m} R_{1}\right) & \leq \gamma^{m} w\left(R_{1}\right)+\gamma\left(R_{1}\right) \sum_{i=0}^{m} \gamma^{i} \\
& \leq \gamma^{m} w\left(R_{0}\right)+\sigma\left(R_{1}\right)\left(\frac{1}{1-\gamma}\right) .
\end{aligned}
$$

Para todo $R \leq R_{1}$ podemos escolher $m$ tal que

$$
\tau^{m} R_{1}<R \leq \tau^{m-1} R_{1}
$$

Logo de (4.55) 


$$
\begin{aligned}
w(R) & \leq w\left(\tau^{m-1} R_{1}\right) \\
& \leq \gamma^{m-1} w\left(R_{0}\right)+\sigma\left(R_{1}\right) \frac{1}{1-\gamma} \\
& \leq \frac{1}{\gamma}\left(\frac{R}{R_{1}}\right)^{\log \gamma / \log \tau} w\left(R_{0}\right)+\gamma\left(R_{1}\right) \frac{1}{1-\gamma}
\end{aligned}
$$

Seja $R_{1}=R_{0}^{1-\mu} R^{\mu}$. De (4.57), tem-se

$$
\begin{aligned}
w(R) & \leq \frac{1}{\gamma}\left(\frac{R}{R_{0}}\right)^{(1-\mu) \frac{\log \gamma}{\log \tau}} w\left(R_{0}\right)+\frac{\sigma\left(R_{0}^{1-\mu} R^{\mu}\right)}{1-\gamma} \\
& \leq C\left(\left(\frac{R}{R_{0}}\right)^{\alpha} w\left(R_{0}\right)+\sigma\left(R^{\mu} R_{0}^{1-\mu}\right)\right)
\end{aligned}
$$

onde $\alpha=(1-\mu) \frac{\log \gamma}{\log \tau}, C=\max \left\{\frac{1}{\gamma}, \frac{1}{1-\gamma}\right\}$, ficando provado o Lema 4.4.1.

Agora, aplicando o Lema 4.4.1 com $\mu$ tal que

$$
(1-\mu) \frac{\log \gamma}{\log \tau}<\mu \sigma
$$

tem-se

$$
\begin{aligned}
w(R) & \leq \frac{1}{\gamma}\left(\frac{R}{R_{0}}\right)^{\mu \tau} w\left(R_{0}\right)+\bar{k}\left(R^{\mu} R_{0}^{1-\mu}\right) \\
& \leq C R^{\mu \sigma}\left(R^{-\mu \sigma} w\left(R_{0}\right)+k\left(R_{0}, f, b, g, d\right)\right) .
\end{aligned}
$$

Logo,

$$
\operatorname{osc}_{B_{R}(y)} u \leq C R^{\mu \sigma}\left(R^{-\mu \sigma} \sup _{B_{0}}|u|+k\right)
$$

ficando provado o Teorema 4.8.

A bibliografia contém 15 livros e artigos, $[1,2,3,4,5,6,7,8,9,10,11$, $12,13,14,15]$. 


\section{Referências bibliográficas}

[1] BREZIS, HAIM. Functional Analysis, Sobolev Spaces and partial Diferential Equations. Springer, USA, 2nd edition, 2011.

[2] EVANS, LAWRENCE C.. Partial Differential Equations. American Mathematical Society, USA, vol.19 edition, 1998.

[3] LADYZHENSKAYA, O. A. AND URAL'TSEVA, N. N.. Linear and Quasilinear Elliptic Equations. Academic Press, New York, 1968.

[4] LADYZHENSKAYA, O. A. , SOLONNIKOV, V. A. AND URAL'TSEVA, N. N.. Linear and Quasilinear Equations of Parabolic Type. Amer. Math. Soc Providence, 1968.

[5] LANDIS, E. M.. Second Order Equations of Elliptic and Parabolic Type. Nauka, Moscow, 1971.

[6] FRIEDMAN, A. . Partial Differential Equations of Parabolic Type. Prentice-Hall, Englewood Cliff, 1964.

[7] NIRENBERG, L.. On nonlinear elliptic partial differential equations and Holder continuity. Commun. Pure Appl. Math., 6, 103-156, 1953.

[8] CORDES, H. O.. Uber die erste Randwertaufgabe bei quasilinearen Differentialgleichungen zweiter ordnung in mehr als zwei Variablen. Math. Ann. 131, 278-312, 1956.

[9] KRYLOV, N. V. AND SAFONOV, M. V.. An estimate of the probability that adiffusion process hits a set of positive measure. Dokl. Anad. Nauk SSSR, 245, No. 1, 18-20, 1979.

[10] AKEKSANDROV, A. D.. Majorization of solution of second-order linear equations. Vestn. Leningr. Univ. Ser. Math. Mekh. Astron., No 1. 5-25, 1966.

[11] GUZMAN, M. . Differential of Integrals in $\mathbb{R}^{n}$. Lecture Notes in Mathematics, No. 481, Springer-Verlag, 1975.

[12] JOHN, F. AND NIRENBERG, L.. On functions of bounded mean oscillation. Comm. Pure Appl. Math. 14, 415-426, 1961. 
[13] MOSER, J.. A new proof of de Giori's theorem concccerning the regularity problem for elliptic differential equations. Comm. Pure Appl. Math. 13, 457-468, 1960.

[14] SAFONOV, M. V.. Harnack's Inequality for elliptic equations and the Holder property of their solutions. Journal of Soviet Mathematics, 21(5), 851-863, 1983.

[15] GILBARG, D. , TRUDINGER, N. S.. Elliptic Partial Differential Equations of Second Order. Springer, 2001. 